ARTICLE

DOI: $10.1038 /$ s41467-018-06224-y

\title{
Silencing of TGF $\beta$ signalling in microglia results in impaired homeostasis
}

\author{
Tanja Zöller ${ }^{1,2}$, Artur Schneider ${ }^{1}$, Christian Kleimeyer ${ }^{1}$, Takahiro Masuda ${ }^{3}$, Phani Sankar Potru ${ }^{2,4}$, \\ Dietmar Pfeifer ${ }^{5}$, Thomas Blank ${ }^{3}$, Marco Prinz $^{3,6}$ \& Björn Spittau ${ }^{1,4}$
}

TGF $\beta 1$ has been implicated in regulating functional aspects of several distinct immune cell populations including central nervous system (CNS) resident microglia. Activation and priming of microglia have been demonstrated to contribute to the progression of neurodegenerative diseases and, thus, underlie stringent control by endogenous regulatory factors including TGF $\beta 1$. Here, we demonstrate that deletion of Tgfbr2 in adult postnatal microglia does neither result in impairment of the microglia-specific gene expression signatures, nor is microglial survival and maintenance affected. Tgfbr2-deficient microglia were characterised by distinct morphological changes and transcriptome analysis using RNAseq revealed that loss of TGF $\beta$ signalling results in upregulation of microglia activation and priming markers. Moreover, protein arrays demonstrated increased secretion of CXCL10 and CCL2 accompanied by activation of immune cell signalling as evidenced by increased phosphorylation of TAK1. Together, these data underline the importance of microglial TGF $\beta$ signalling to regulate microglia adaptive changes.

\footnotetext{
${ }^{1}$ Institute for Anatomy and Cell Biology, Department of Molecular Embryology, Faculty of Medicine, University of Freiburg, 79104 Freiburg, Germany. ${ }^{2}$ Faculty of Biology, University of Freiburg, 79104 Freiburg, Germany. ${ }^{3}$ Institute of Neuropathology, Medical Center-University of Freiburg, Faculty of Medicine, University of Freiburg, 79106 Freiburg, Germany. ${ }^{4}$ Institute of Anatomy, University of Rostock, 18057 Rostock, Germany. ${ }^{5}$ Department of Hematology/Oncology, Faculty of Medicine, University of Freiburg, 79106 Freiburg, Germany. ${ }^{6}$ BIOSS Centre for Biological Signalling Studies, University of Freiburg, 79104 Freiburg, Germany. Correspondence and requests for materials should be addressed to B.S. (email: bjoern.spittau@med.uni-rostock.de)
} 
M icroglia represent the specific immune cell population of the central nervous system (CNS), are involved in physiological CNS functions and further participate in the development, progression as well as the resolution of pathological conditions ${ }^{1,2}$. As part of the innate immune system, microglia are able to sense pathogen-associated molecular patterns and danger-associated molecular patterns resulting in acute microglia reactivity ${ }^{3}$. However, chronic stimulation of microglia has been shown to induce a hyper-reactive phenotype which is referred to as primed and which is characterised by prolonged and exacerbated reactions. Primed microglia have been detected in several neurodegenerative diseases and during aging of microglia ${ }^{4,5}$. Recent studies have demonstrated that microglia derive from primitive macrophage precursors ${ }^{6}$ involving PU.1- as well as IRF8-dependent signalling pathways ${ }^{7}$. Homing of microglia towards the CNS parenchyma is mediated by the CSFR1/IL34 receptor/ligand pair and occurs during mid and late embryonic development ${ }^{6,8}$. Microglia develop a specific gene expression signature in the CNS parenchyma, which is distinct from the molecular signature of monocytes and monocytederived macrophages providing the possibility to segregate these distinct cell populations from each other ${ }^{9,10}$. The cell-surface protein TMEM119 was recently characterised as a microgliaspecific marker in mouse and human and has been further used to determine that mouse microglia adopt their specific gene expression signature within the first two postnatal weeks ${ }^{11}$. This distinct microglial molecular signature was absent in mice with a CNS-specific deletion of transforming growth factor beta 1 (TGF $\beta 1)$ and mutant mice displayed a reduction in microglia numbers which was observed from postnatal day 20 into adulthood. Moreover, postnatal development and survival of these mutant mice were impaired suggesting the contribution of mechanisms outside of the CNS to the phenotype development ${ }^{12}$. Nevertheless, it remains elusive whether the observed effects are dependent on microglial TGF $\beta$ signalling or whether secondary effects mediated by TGF $\beta$-sensitive CNS cell populations, such as neurons, astrocytes or oligodendrocytes are responsible for the microglial phenotype reported by Butovsky et al. ${ }^{12}$. However, the above-mentioned results identified TGF $\beta 1$ as an essential endogenous factor to promote microglial maturation as a prerequisite for adequate microglia functions in the adult CNS. TGF $\beta 1$ has previously been described as a potent immunoregulatory factor for microglia in vivo and in vitro ${ }^{13-15}$.

TGF $\beta$-signalling is propagated by binding of TGF $\beta$ to the TGF $\beta$ receptor type II (Tgfbr2) that phosphorylates the TGF $\beta$ receptor type I (Tgfbr1), thus, triggering the kinase activity of Tgfbr $1^{16,17}$. Receptor-associated signalling mediators Smad2 and Smad3 are the primary phosphorylation targets of Tgfbr1 and form a complex with the common mediator Smad4 to translocate to the nucleus and to further regulate the expression of TGF $\beta$ target genes ${ }^{18}$.

Unfortunately, the postnatal lethality of TGF $\beta 1$-deficient mice due to severe systemic immune effects limited the analysis of the TGF $\beta 1$-mediated regulation of microglia activation in adult mice ${ }^{19}$. Moreover, lethal embryonic phenotypes of Tgfbr2- and Smad4-deficient mice restricted the evaluation of TGF $\beta$-signalling in adult microglia ${ }^{20,21}$. Taking advantage of the unique microglial nature and the development of new genetic tools ${ }^{22}$ we addressed the role of TGF $\beta$-signalling during microglial maintenance and quiescence in adult mice.

Here, we used $C \times 3 \operatorname{cr} 1 C r e^{E R T 2}$ mice to induce the conditional deletion of the ligand-binding receptor $T g f b r 2$ in adult microglia. Our data indicate that postnatal silencing of microglial TGF $\beta$ signalling impairs microglia quiescence rather than compromising microglia maintenance and survival in vivo. Moreover, RNAseq-based gene expression analysis revealed that silencing
TGF $\beta$ signalling in adult microglia does not affect the postnatally induced microglia-specific gene expression pattern but results in microglia activation and priming. We further demonstrate that increased TAK1 phosphorylation in Tgfbr2-deficient microglia and enhanced cytokine release could be detected suggesting the loss of microglial quiescence. Overall, these data underline the importance of TGF $\beta$-signalling for regulation of microglia activation and further indicate that the maturation and maintenance functions of TGF $\beta 1$ take place at earlier postnatal stages. Finally, the mutant mouse line introduced in the present study constitute a powerful tool to analyse the contribution of microglial TGF $\beta$ signalling in neuropathological conditions associated with microglia activation.

\section{Results}

Induction of postnatal $T g f b r 2$ deletion in microglia. In order to evaluate the importance of TGF $\beta$-signalling for postnatal microglia survival and maintenance, a conditional microglia/macrophage-specific mutant mouse line for $T g f b r 2$ was generated by crossing $C \times 3 c r 1^{C r e E R T 2}$ mice to mice carrying loxP ${ }^{-}$site-flanked (floxed) alleles of Tgfbr2 to obtain Cx3cr1 CreERT2:Tgfbr $2^{f l / f l}$ mice. The ROSA26-YFP (R26-yfp) mouse line was additionally crossed into the $C x 3 \mathrm{cr} 1^{C r e E R T 2}: T g f b r 2^{f l f l}$ line, resulting in the transgenic line $C \times 3 c r 1^{C r e E R T 2}: R 26-y f p$,Tgfbr $2^{f l f l}$ (Fig. 1a). Tamoxifen (TAM)induced recombination in vivo (Supplementary Fig. 1A) and in vitro (Supplementary Fig. 1B) resulted in the deletion of the floxed Exons $2 / 3$ of the Tgfbr 2 gene which code for the ligandbinding domain of the receptor (Fig. 1b). Sequencing of recombined primary microglia and $\mathrm{qPCR}$ after recombination in vitro and in vivo using exon-specific primers revealed deletion of Exons $2 / 3$ in $C \times 3 c r 1^{C r e E R T 2}: R 26-y f p, T g f b r 2^{f l / f l}$ microglia (Supplementary Fig. 1). Noteworthy, lack of Exons $2 / 3$ did not affect the reading frame of $T g f b r 2$ and, thus, recombined microglia expressed a dominant negative form of Tgfbr2 with normal serine/threonine kinase activity but abrogated ligand-binding capacity (Fig. 1b). In order to exclude phenotypic effects obtained due to $C x 3 \mathrm{cr} 1$ haploinsufficiency in $C x 3 c r 1^{\text {CreERT2 }}$ mice or TAM itself, additional control groups were used throughout this study. $C \times 3 c r 1^{+/+}: R 26-$ $y f p, T g f b r 2^{f l / f l}$ mice treated with TAM (+/+TAM) and CX3cr1 ${ }^{C r E E R T 2}: R 26-y f p, T g f b r 2^{f l / f l}$ mice treated with corn oil (cre/ +OIL) were considered as controls, whereas Cx3cr1 ${ }^{C r e E R T 2}: R 26-$ $y f p, T g f b r 2^{f l f l}$ mice treated with TAM (cre/+TAM) are referred to as recombined knockout mice in vivo. Similar control groups were used for in vitro experiments where ethanol served as the solvent for TAM $(+/+\mathrm{EtOH},+/+\mathrm{TAM}, \mathrm{Cre} /+\mathrm{EtOH}, \mathrm{Cre} /+$ TAM). In order to test the recombination efficacy detection of stable YFP expression in microglia was assessed in vitro (Fig. 1c) and in vivo (Fig. 1d), as also described recently ${ }^{22}$. In both conditions, recombination rates of approximately $80 \%$ of $\mathrm{F} 4 / 80^{+}$microglia were achieved (Fig. 1c, in vitro $79.77 \pm 6.69 \% \mathrm{YFP}^{+}$cells, Fig. $1 \mathrm{~d}$, in vivo $82.28 \pm 18.53 \% \mathrm{YFP}^{+}$cells). Recombination efficacy was further analysed in $\mathrm{CD} 115^{+} /$Ly6 $\mathrm{C}^{\text {low }}$ blood monocytes 1 week and 4 weeks after TAM injections to monitor the turnover of peripheral $\mathrm{Cx} 3 \mathrm{crl}^{+}$myeloid cells. While a time-dependent reduction of $\mathrm{YFP}^{+}$blood monocytes from $43 \%$ (1 week) to $9.8 \%$ (4 weeks) could be detected, the numbers of $\mathrm{F} 4 / 80^{+} / \mathrm{YFP}^{+}$ microglia remained stable (Supplementary Fig. 2). Next, we validated the insensitivity of recombined microglia towards TGF $\beta 1$. Whereas recombinant TGF $\beta 1(5 \mathrm{ng} / \mathrm{ml}, 2 \mathrm{~h})$ induced phosphorylation and nuclear translocation of SMAD2 in control microglia, no SMAD2 phosphorylation and nuclear translocation were detected in mutant microglia (Fig. 1e, f). Together, these data demonstrate that TAM-induced recombination resulted in the stable deletion of Tgfbr2 Exons $2 / 3$ and abrogation of SMAD2mediated TGF $\beta 1$ signal transduction in mutant microglia. 
a

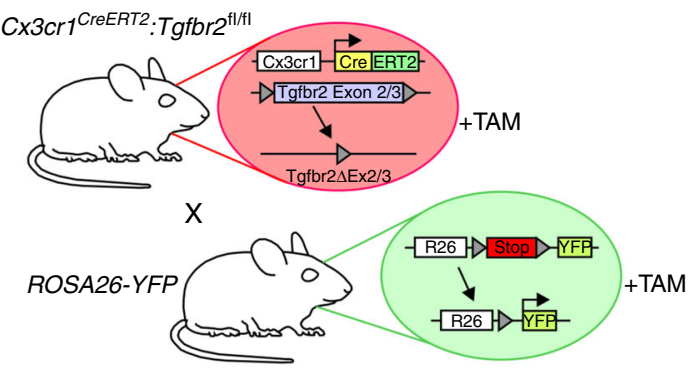

b

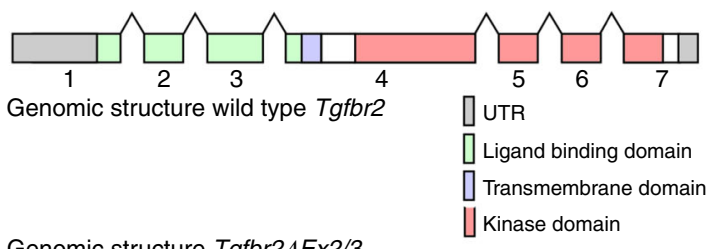

Genomic structure Tgfbr24Ex2/3
C
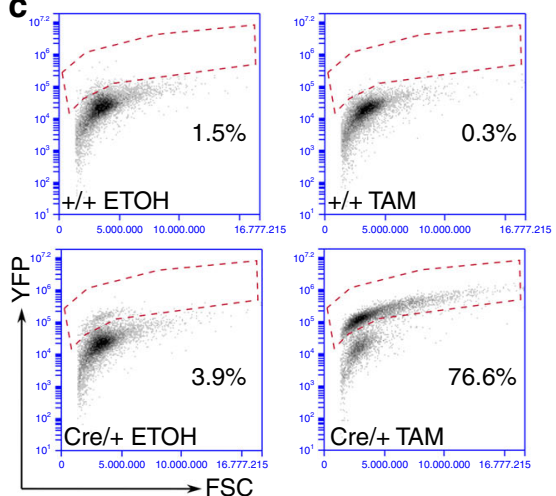

e
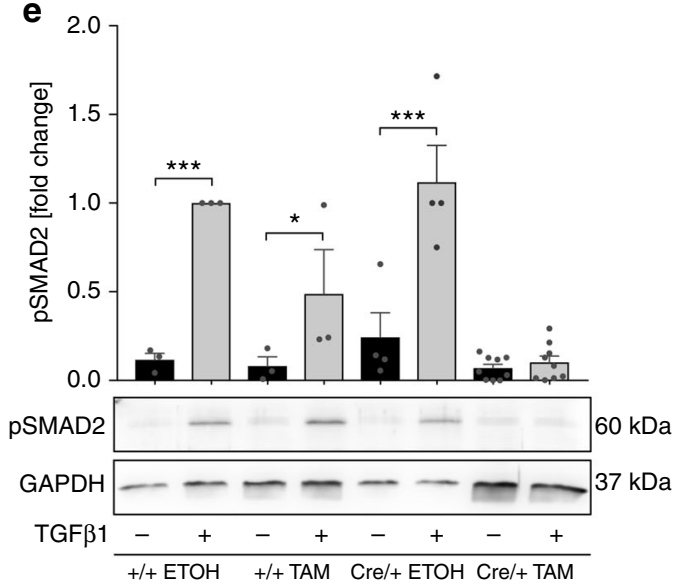
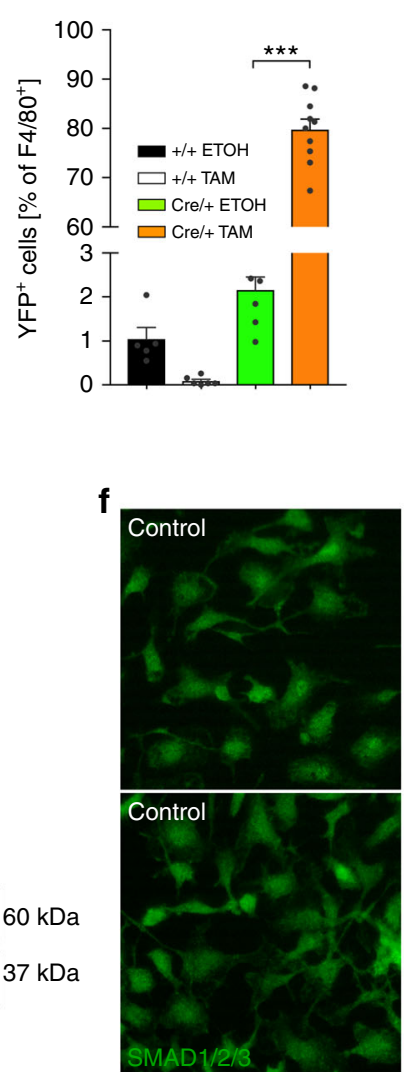

d
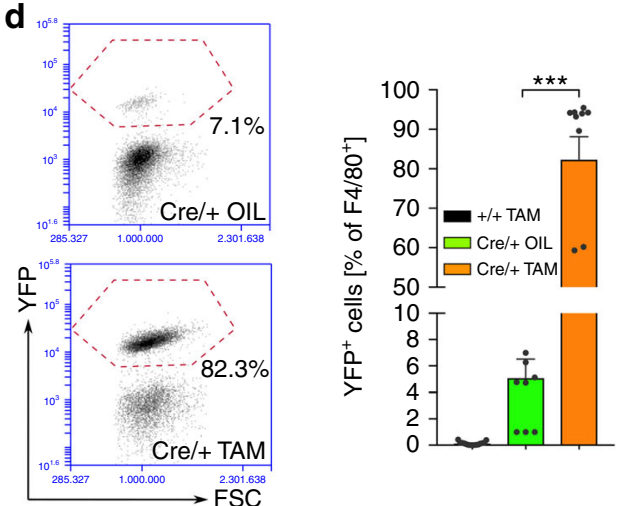

Fig. 1 Successful microglia-specific knockout of Tgfbr2 in vitro and in vivo. a Scheme illustrating breeding strategy to obtain Cx3cr7 CreERT2:R26-yfp,Tgfbr2fl/fl mice for TAM-induced deletion of Tgfbr2 Exons 2/3 and induction of YFP reporter gene expression. $\mathbf{b}$ Genomic structure of wild-type and mutant Tgfbr2 after TAM-induced recombination. c Flow cytometric analysis of microglia 3 days after TAM-induced recombination in vitro for expression of YFP. Data are presented as mean $\pm \mathrm{SEM}(+/+\mathrm{EtOH} n=5,+/+\mathrm{TAM} n=6, \mathrm{Cre} /+\mathrm{EtOH} n=7, \mathrm{Cre} /+\mathrm{TAM} n=10) . P$ values derived from one-way ANOVA are ${ }^{\star \star \star} p<$ 0.001. d Flow cytometric analysis of YFP reporter gene expression 4 weeks after recombination in vivo. Data are given as means \pm SEM $(+/+\mathrm{TAM} n=12$, $\mathrm{Cre} /+\mathrm{OIL} n=8, \mathrm{Cre} /+\mathrm{TAM} n=10)$. P values derived from one-way ANOVA are ${ }^{\star \star \star} p<0.001$. e Primary microglia from newborn $C \times 3 c r 7{ }^{C}$ reERT2:R26-yfp, Tgfbr $2^{f l / f l}$ were recombined in vitro. Control $(+/+\mathrm{EtOH},+/+\mathrm{TAM}, \mathrm{Cre} /+\mathrm{EtOH})$ and knockout (Cre/+ TAM) samples were treated with TGF $\beta 1$ (5 ng/ $\mathrm{ml})$ for $2 \mathrm{~h}$. Quantification of SMAD2 phosphorylation (pSMAD2) after densitometric evaluation and GAPDH normalisation $(+/+$ EtOH $n=3,+/+$ TAM $n=3, \mathrm{Cre} /+\mathrm{EtOH} n=4, \mathrm{Cre} /+\mathrm{TAM} n=9)$. Data are given as fold changes compared to $+/+\mathrm{EtOH}$-treated samples. $P$ values derived from one-sample $t$ tests are ${ }^{\star} p<0.05$ and ${ }^{* \star *} p<0.001$. f Nuclear translocation of SMAD2 was determined in primary microglia after treatment with TGF $\beta 1$ ( $\left.5 \mathrm{ng} / \mu \mathrm{l}\right)$ for $2 \mathrm{~h}$. Scale bar represents $50 \mu \mathrm{m}$. Data are given as means \pm SEM from three independent experiments. $P$ values derived from one-way ANOVA are ${ }^{\star \star \star} p<0.001$

Tgfbr2 is dispensable for microglia survival and maintenance. Body weight and survival of knockout mice as well as impaired microglia maintenance has been described in mice lacking expression of TGF $\beta 1$ in the $\mathrm{CNS}^{12}$. In order to evaluate the contribution of microglial TGF $\beta$ signalling to these phenotypes, body weight and survival of $C x 3 c r 1^{C r e E R T 2}: R 26-y f p, T g f b r 2^{f l / f l}$ mice was analysed. Interestingly, no impairment of body weight increase up to 160 days after recombination could be observed (Fig. 2a). Moreover, mouse survival as analysed up to 315 days after recombination was not affected (Fig. 2b). Quantifications of cortical Iba1 ${ }^{+}$microglia were performed early (60 days) and late after TAM-induced recombination (150 days). At both time points, no significant differences in microglia numbers between controls and knockout samples could be detected (Fig. 2c). However, distinct morphological changes of microglia could be observed after TAM-induced recombination (Fig. 2d, e). In order to address whether neurons numbers were affected by loss of microglial TGF $\beta$ signalling, cortical $\mathrm{NeuN}^{+}$cells were quantified. As depicted in Fig. 2f, g, normal distribution of cortical neurons in layers 3-5 could be observed 60 days after recombination. 
a

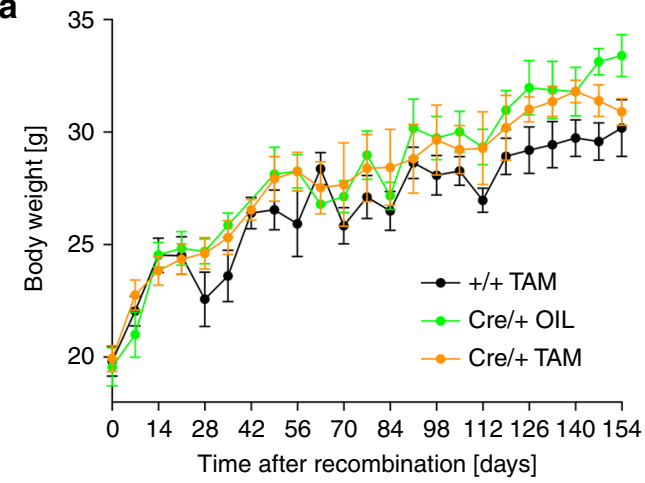

b

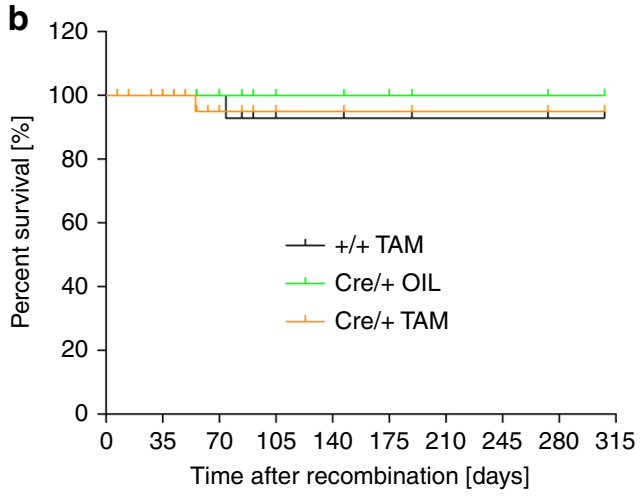

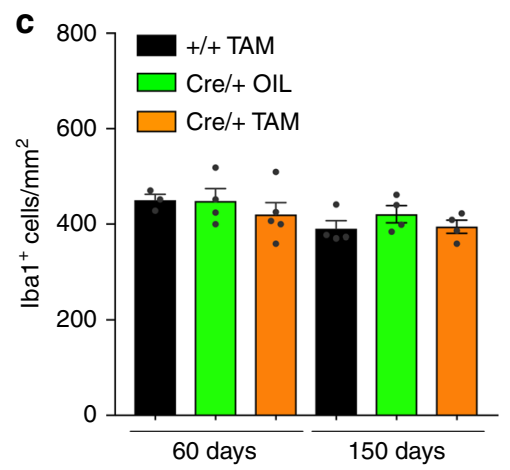
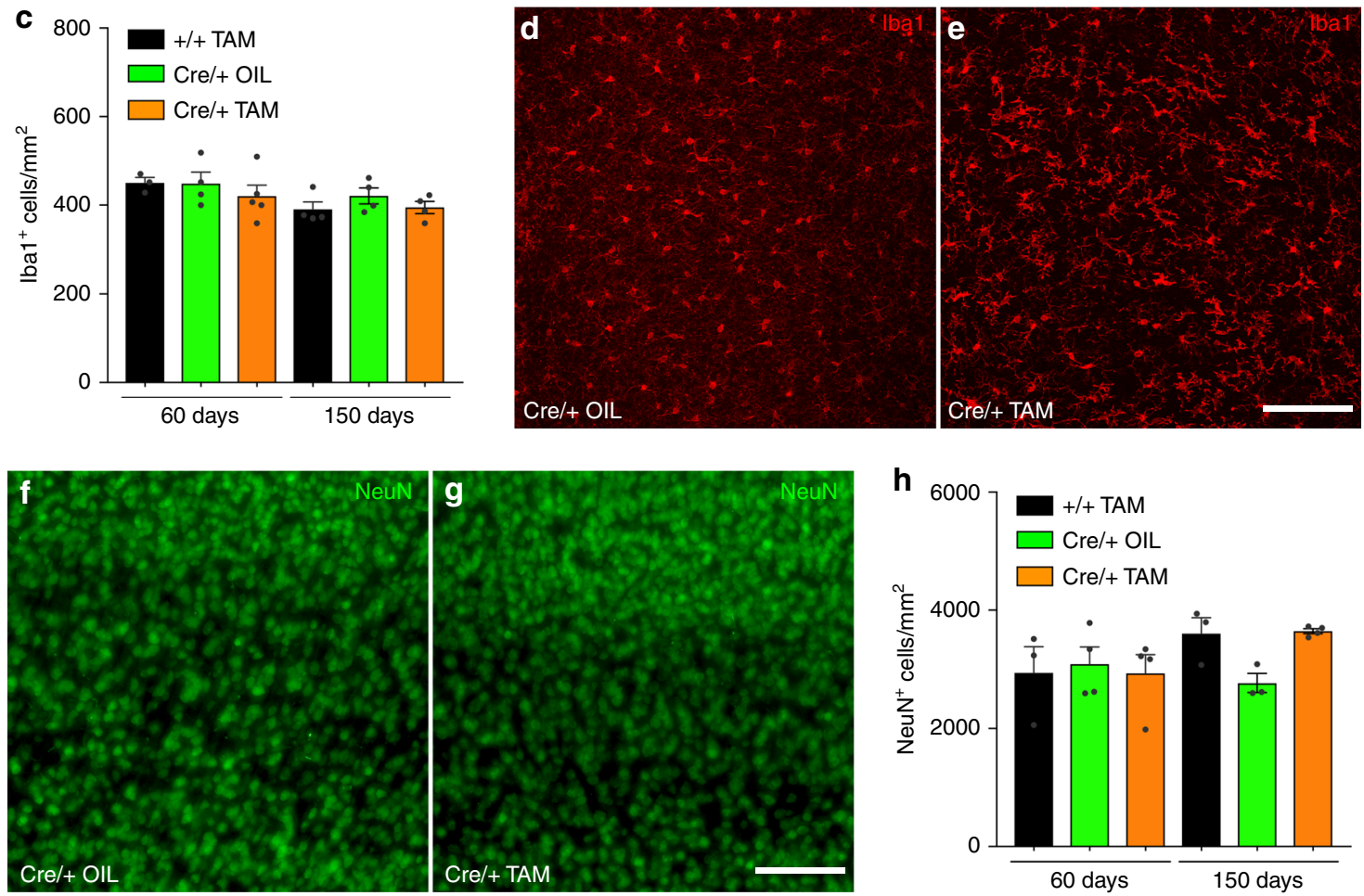

Fig. 2 Normal mouse survival and no impairment of microglia maintenance after microglia-specific knockout of Tgfbr2. a Body weight increase after TAMinduced recombination at 6-8 weeks of age (timepoint 0$)$. Weights were measured weekly in control $(+/+\mathrm{TAM}, \mathrm{Cre} /+\mathrm{OIL})$ and knockout $(\mathrm{Cre} /+$ TAM) animals. Data points indicate mean \pm SEM $(n=3-18$ mice). b Kaplan-Meier survival curves of control and knockout mice after TAM-induced recombination in vivo. c Microglia numbers given as $\mathrm{lba} 1^{+}$cells $/ \mathrm{mm}^{2}$ were obtained from three cortical fields per animal. Iba $1^{+}$microglia were analysed 60 days and 150 days after TAM-induced recombination. $\mathbf{d}$ lba1 immunostainings from the cortices of control mice (Cre/+ OIL) and e knockout mice $(\mathrm{Cre} /+\mathrm{TAM})$ after 60 days. Cortical neuron $\left(\mathrm{NeuN}^{+}\right)$distributions in $\mathbf{f}$ control mice and $\mathbf{g}$ knockout mice 60 days after TAM-induced recombination. Scale bars represent $100 \mu \mathrm{m}$. h Quantification of cortical neuron numbers in control (+/+ TAM, Cre/+ OIL) and knockout (Cre/+ TAM) animals revealed no significant changes. Data are given as means \pm SEM from at least three animals per genotype and timepoint

Moreover, no significant changes in neuron numbers were detected 60 days and 150 days after TAM-induced recombination (Fig. 2h). Collectively, these data indicate that TGF $\beta$ signalling is dispensable for postnatal survival and maintenance of adult microglia and does not affect the survival of cortical neurons.

Tgfbr2 regulates microglia reactivity. Since TGF $\beta 1$ has been demonstrated to regulate microglial homeostasis ${ }^{15}$, we further addressed whether microglia in $C \times 3 c r 1^{C r e E R T 2}: R 26-y f p, T g f b r 2^{f l / f l}$ mice displayed an activation phenotype. First, a detailed and quantitative morphometric analysis of $\mathrm{Iba}^{+}$microglia lacking Tgfbr 2 has been performed. Control microglia $(+/+$ TAM and Cre/+ OIL) showed typical ramified morphologies, such as round to spindle-shaped somata and distinct arborisation patterns with finely delineated processes (Fig. 3a, b). In contrast, $\mathrm{YFP}^{+} \mathrm{Tgfbr} 2$ knockout microglia (Cre/+ TAM) presented a heterogeneous morphology, which could be divided into three distinct subentities further referred to as "ramified", "bushy" and "hypertrophied" microglia (Fig. 3c, d). First, the prevalence of these different microglia morphologies has been determined in all genotypes analysed. Whereas the number of ramified microglia was significantly reduced in Cre/+ TAM microglia, numbers of hypertrophied and bushy microglia were increased. Noteworthy, hypertrophied as well as bushy microglia could rarely be detected in cortices from control mice (Fig. 3e). Three-dimensional reconstructions (Fig. 3f) revealed that the filament length was reduced in hypertrophied and to a lesser extent in bushy microglia (Fig. $3 \mathrm{~g}$ ). The area covered by microglia processes was significantly reduced in both bushy and hypertrophied microglia 

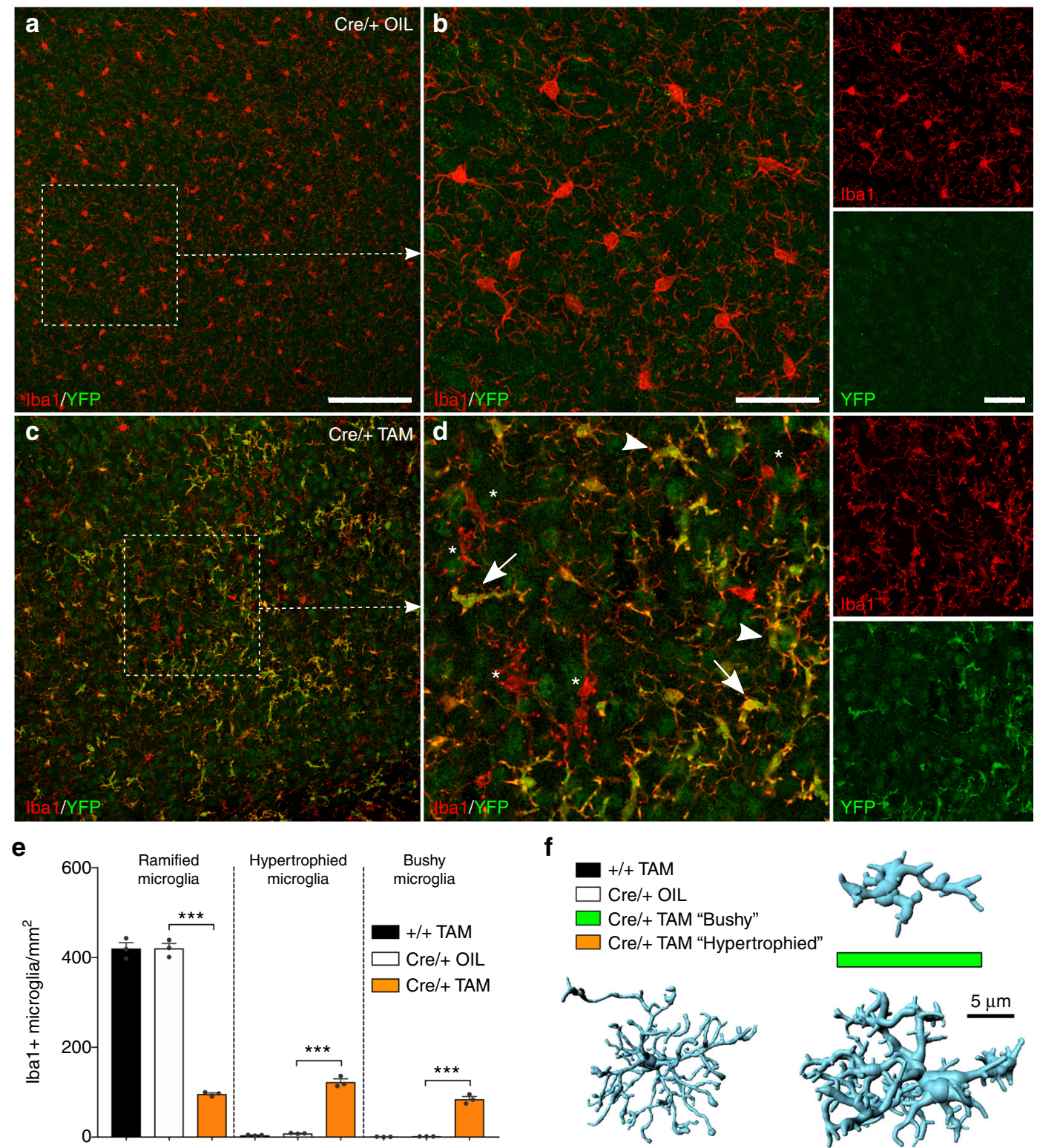

f
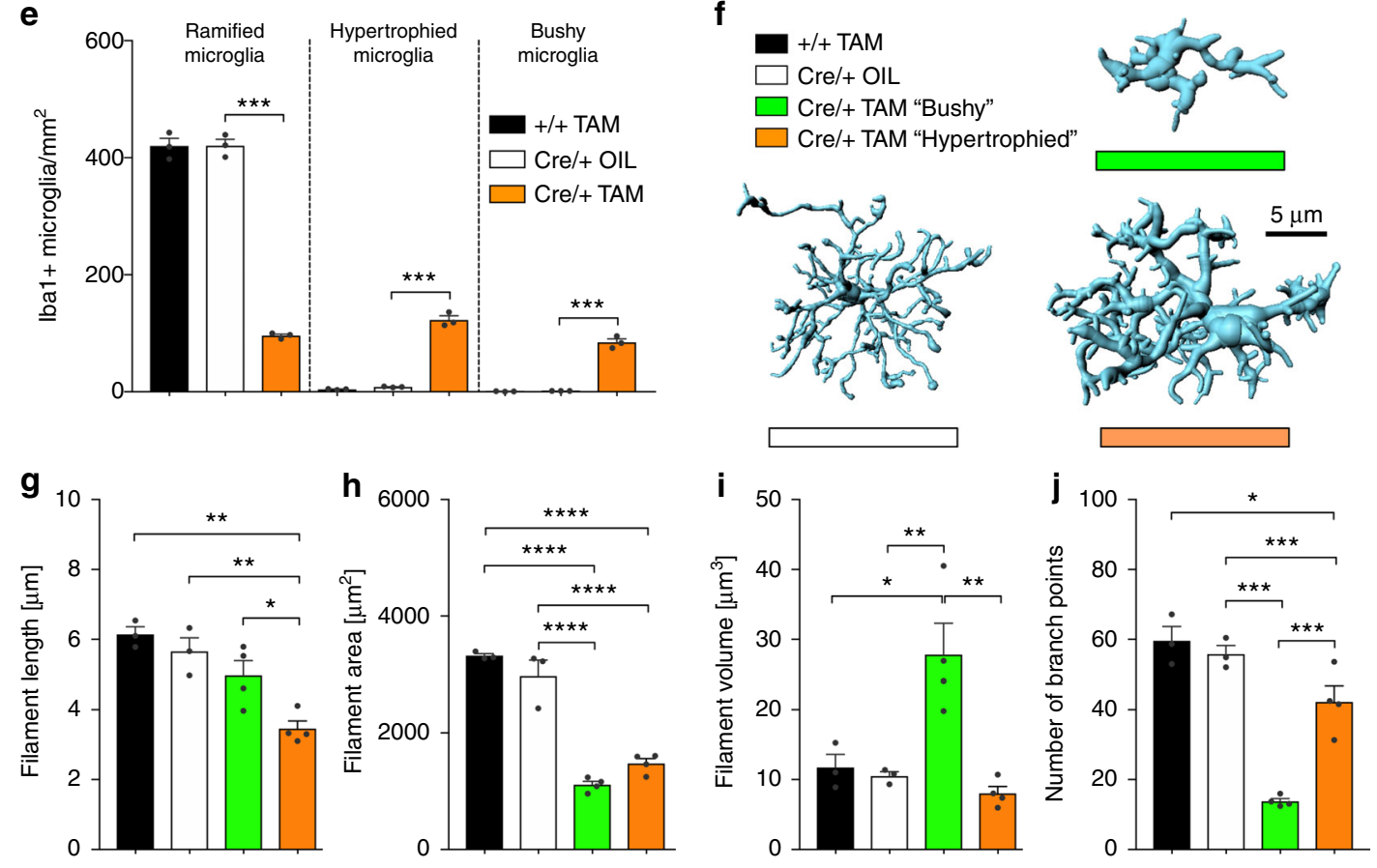

compared to controls (Fig. 3h). Filament volume was strongly increased in bushy microglia (Fig. 3i) and the numbers of branching points were dramatically reduced in bushy microglia and to a minor extent in hypertrophied microglia (Fig. 3j). Numbers of terminal points and numbers of segments (Supplementary Fig. 3) further demonstrate that Tgfbr2-deficient microglia display distinct morphological changes in vivo. Since morphology changes can be taken as a proxy for different

microglia activation states $^{23}$, we further analysed the gene expression patterns of Tgfbr2-knockout microglia by using RNAseq. Adult isolated microglia from tamoxifen-injected $C \times 3 c r 1^{C r e E R T 2}: R 26-y f p, T g f b r f^{f l / f l}$ and $C \times 3 c r 1^{+/+}: R 26-y f p, T g f b r 2^{f l}$ $f l$ mice were sorted as $\mathrm{YFP}^{+} / \mathrm{CD} 45^{\text {low }}$ and $\mathrm{CD} 11 \mathrm{~b}^{+} / \mathrm{CD} 45^{\text {low }}$ respectively (Fig. 4a). Interestingly, comparative RNAseq analysis revealed that the expression of microglia-specific genes such as Csf1r, Cx3cr1, Fcrls, Olfml3, P2ry12, Sall1, SiglecH or, Tmem119 


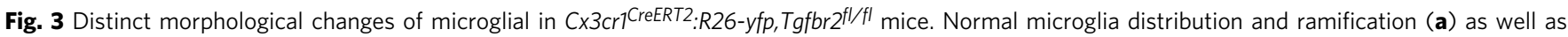
normal microglia morphology and no YFP ${ }^{+}$cells in control mice 4 weeks after TAM injections (b). Distinct heterogenous distribution (c) and morphological changes of $\mathrm{YFP}^{+} / \mathrm{Iba}^{+}$microglia 4 weeks after TAM-induced deletion of Tgfbr2 (d). Based on their morphology, microglia in cre/+ TAM knockout mice were divided into "normally ramified", "bushy", and "hypertrophied". White arrowheads mark hypertrophied microglia, white arrows mark bushy microglia, and white asterisks mark YFP-/lba1+ nonrecombined microglia. Scale bars represent $100 \mu \mathrm{m}$ (a, c) and $25 \mu \mathrm{m}$ (b, d). e Prevalence of normally ramified, bushy, and hypertrophied microglia in different experimental groups. Data are given as means \pm SEM from at least three animals per genotype 4 weeks after recombination. $P$ values derived from one-way ANOVA are ${ }^{\star \star \star} p<0.001$. $\mathbf{f}$ Imaris (Bitplane)-based three-dimensional reconstructions of representative $\mathrm{lba} 1^{+}$microglia from $\mathrm{C} \times 3 \mathrm{cr} 7^{\mathrm{CreERT2}}: R 26-\mathrm{yfp}, \mathrm{Tgfbr} 2^{\mathrm{fl} / \mathrm{fl}}$ mice. Imaris-based automated quantification of microglial filament length (g), filament area (h), filament volume (i), and numbers of branch points $(\mathbf{j})$. Data are presented as means \pm SEM of 20 cells in $3-4$ animals per group. $P$ values derived from one-way ANOVA are ${ }^{\star} p<0.05,{ }^{\star \star} p<0.01,{ }^{\star \star \star} p<0.001$ and ${ }^{\star \star \star \star} p<0.0001$

was not impaired after TAM-induced deletion of Tgfbr2 suggesting that TGF $\beta$ signalling is dispensable for maintenance of the molecular microglia signature in adult mice (Fig. 4b). However, analysis of differentially regulated genes in Tgfbr2-deficient microglia revealed 51 upregulated and 4 downregulated genes (with $<2$-fold change [ $1 \log 2$ ratio] and $p<0.05$; Fig. $4 c$ ). Functional categorisation using the DAVID bioinformatics database ${ }^{24}$ for GO term enrichment analysis of biological process (Fig. 4d) molecular function (Fig. 4e) and KEGG pathway enrichment (Fig. 4f) indicate that Tgfbr2-deficient microglia are immunologically active. Cellular compartment enrichment analysis revealed increased expression of membrane components including Ms4a4a, Ms4a14, Ms4a7, Cd74, Cd52, Mrc1, and $A x l$ as well as MHCII-related genes H2-Aa, H2-Ab1, H2-Q5, and H2-Q7. Moreover, increased expression of secreted molecules $C p, C c l 7$, Pf4, Tnf, and Tnfsf 8 was detected in mutant microglia (Fig. $4 \mathrm{~g}$ ). Taken together, these data indicate that loss of TGF $\beta$ signalling in adult microglia results in a primed and activated microglia phenotype but does not impair the maintenance of the microgliaspecific gene expression signature in vivo.

Increased activation marker expression in mutant microglia. To address the expression of microglia activation markers in mutant mice, adult acutely isolated microglia were used for flow cytometry analysis at least 3 weeks after tamoxifen-induced recombination. Cells were gated for F4/80 and stained for activation markers CD86, CD206, CD36, and MHCII, which were chosen based on a screening for TGF $\beta 1$-regulated genes in primary microglia, where CD86, CD206, CD36 were downregulated after TGF $\beta$ treatment and upregulated after abrogation of TGF $\beta$ signalling by application of a Tgfbr1 inhibitor (Supplementary Fig. 4). In $C \times 3 c r 1^{C r e E R T 2}: R 26-y f p, T g f b r 2^{f l f l}$ knockout mice, the percentages of $C D 86^{\text {high }}$ and $C D 206^{+}$cells significantly increased in adult isolated microglia. No significant changes were observed for surface expression levels of CD36 and MHCII in Tgfbr2deficient microglia (Fig. 5a, b). Analogue to in vivo experiments, primary microglia were recombined in vitro and used for flow cytometry analysis. Deletion of Tgfbr2 in Cx3cr1 CreERT2:R26-yfp, Tgfbr $2^{f l f l}$ microglia resulted in dramatic increases of all activation markers analysed. The percentages of CD86 high $(83.1 \%)$, CD206 $+(58.2 \%), \mathrm{CD}^{\text {high }}$ (91.9\%), and MHCII ${ }^{\text {high }}$ (67.8\%) microglia in knockout samples were strongly increased with high significancies (Fig. 5c, d). We further analysed whether microglia express different activation markers at the same time. As shown in Fig. 5e, Tgfbr2-deficient $\mathrm{CD} 86^{+}$microglia were also positive for CD206 (64.5\%) and CD36 (97.1\%) and the majority of CD206 ${ }^{+}$ microglia were double-positive for CD36 (80.0\%) indicating that microglia with abrogated TGF $\beta$ signalling increase expression of CD86, CD206 as well as CD36 (Fig. 5e). Interestingly, the observed increase in microglia surface activation markers was more pronounced in vitro suggesting that primary microglia in vitro are preactivated and/or differ in their sensitivity towards TGF $\beta$.
Cytokine release and TAK1 activation in Tgfbr2-KO microglia. In vitro recombined microglia were used to analyse signalling pathways involved in inflammatory responses and supernatants were collected to assess the release of chemokines and cytokines (Fig. 6a). Tgfbr2-deficient microglia obtained from $C \times 3 \mathrm{cr} 1^{\mathrm{CreERT} 2}$ : $R 26-y f p, T g f b r 2^{f l / f l}$ mice showed a significant increase of TAK1 phosphorylation and a slight but not significant increase in p38 MAPK phosphorylation (Fig. 6b). Moreover, the secretion of CCL2 and CXCL10 was significantly increased whereas the release of CCL3 was decreased in Tgfbr2-deficient microglia. These observations are in accordance with CXCL10 and CCL2 expression after treatment of primary wild-type microglia with recombinant TGF $\beta 1$, which resulted in downregulation of $\mathrm{Cxcl10}$ and $\mathrm{Ccl} 2$ and subsequent decrease in CXCL10 (Fig. 6c, Supplementary Fig. 5). It has been recently demonstrated that TGF $\beta 1$ is sufficient to promote microglial quiescence and inhibit IFN $\gamma$ induced microglia activation ${ }^{15,25}$. In order to address whether these anti-inflammatory effects of TGF $\beta 1$ are compromised, transgenic microglia from $C x 3 c r 1^{C r e E R T 2}: R 26-y f p, T g f b r 2^{f l / f l}$ mice have been used to prepare primary microglia cultures for in vitro recombination. Expression of $i N o s$ and Tnf $\alpha$ was analysed after microglia activation with IFN $\gamma$. Tgfbr2-deficient microglia displayed a similar activation response upon IFN $\gamma$ treatment as control microglia. However, TGF $\beta 1$ treatment did not result in inhibition of IFN $\gamma$-induced activation of Tgfbr2-deficient microglia (Fig. 6d, e). In summary, these data demonstrate that loss of TGF $\beta$ signalling in microglia results in increased immune cell signalling as evidenced by TAK1 phosphorylation and the release of proinflammatory cytokines CCL2 and CXCL10. Moreover, silencing TGF $\beta$ signalling renders microglia insensitive for TGF $\beta 1$-mediated inhibition of IFN $\gamma$-induced microglia activation.

\section{Discussion}

It has become clear that microglia activation contributes to the neuronal loss in neurodegenerative pathologies including $\mathrm{Alz}$ heimer's disease (AD) and Parkinson's disease (PD) ${ }^{5,26}$. However, during development and maintenance of the CNS microglia are constantly involved in supporting physiological functions such as synaptic pruning 27,28 , synapse formation during learning ${ }^{29}$, support of axonal outgrowth ${ }^{30}$ as well as maintenance and survival-promoting effects for neurons ${ }^{31}$. In order to fulfil these physiological functions, microglia need to establish a distinct gene expression pattern which clearly distinguishes them from macrophages ${ }^{10}$. This microglia maturation has recently been demonstrated to take place during the first postnatal weeks in mice and is characterised by the expression of microglia-specific genes such as P2ry12, Fcrls, Tmem119, Cx3cr1, Csf1r, Sall1, Siglech, and Olfml3 10,11 . It is only partially understood which endogenous factors are involved in guiding postnatal microglia maturation. However, TGF $\beta 1$ has been identified as one important player for microglia maturation. In order to overcome the lethal phenotype of TGF $\beta 1$ knockout mice, which develop a 
a

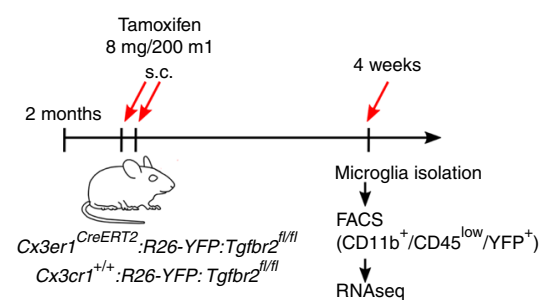

b

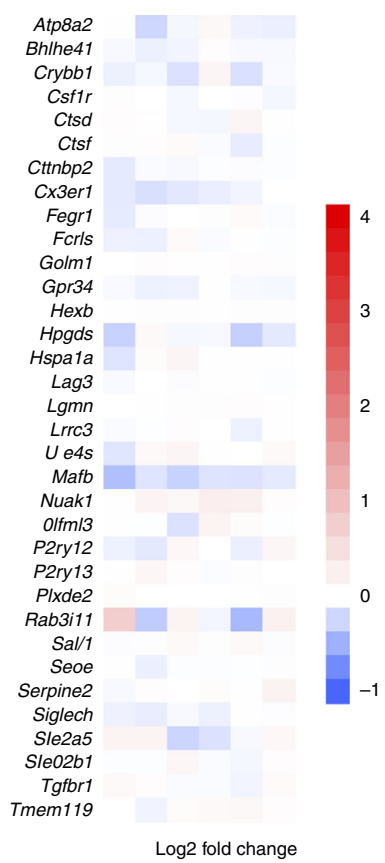

$\mathbf{f}$

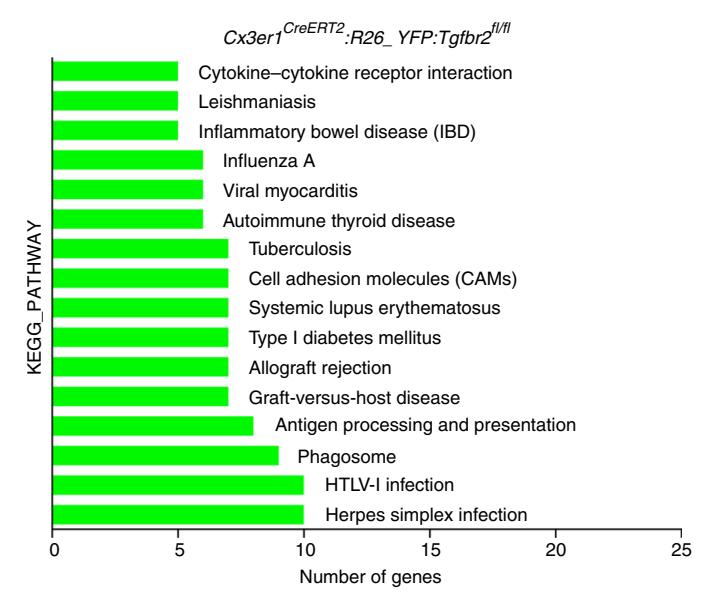

C

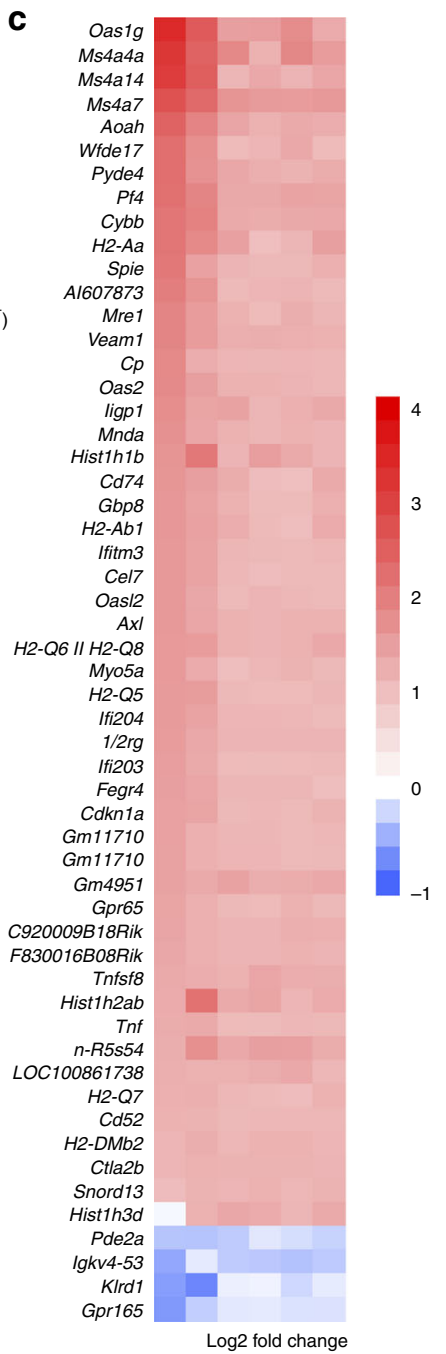

d

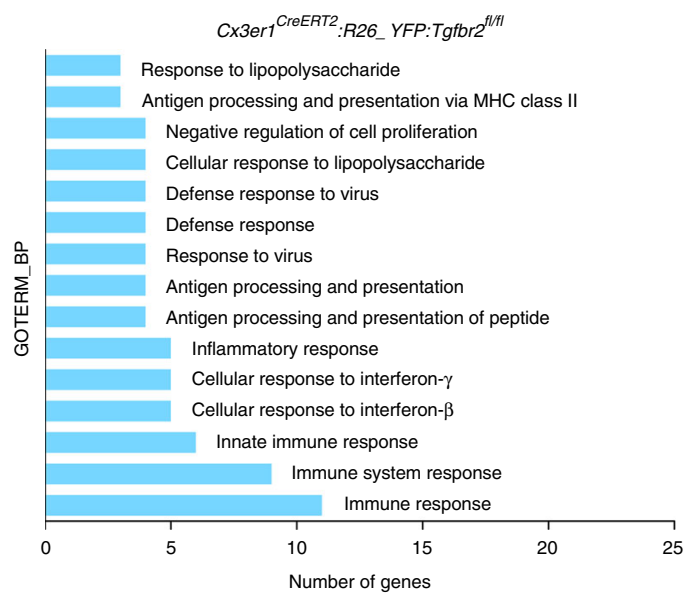

e

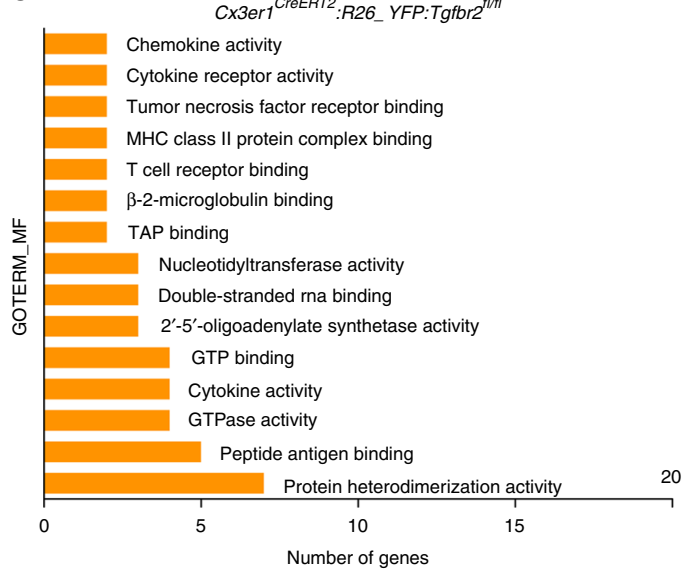

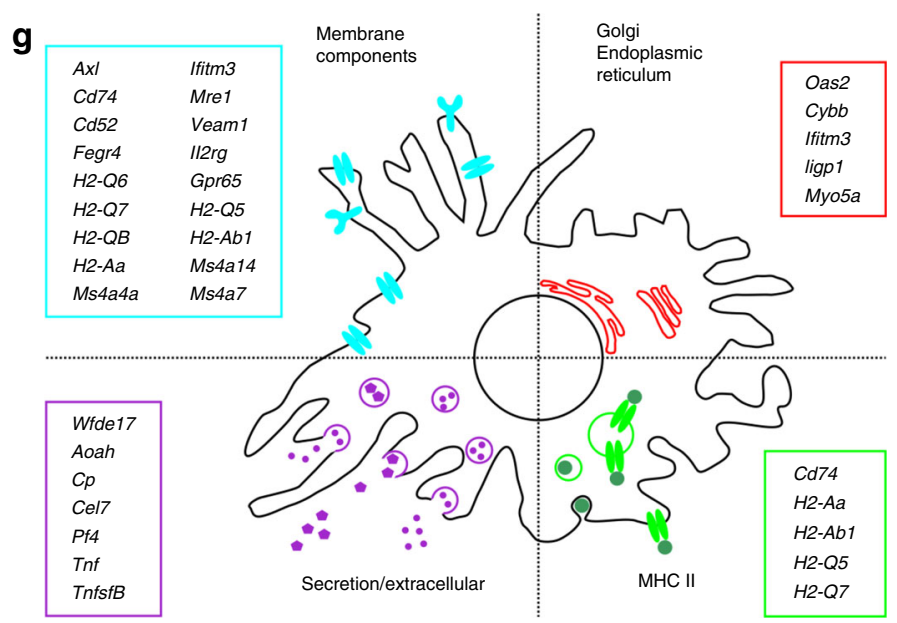

Fig. 4 Gene expression pattern of Tgfbr2-deficient microglia indicates microglia activation and increased immune responses. a A timeline depicting TAMtreatment and microglia isolation/sorting for RNA isolation. b Heatmap analysis of RNAseq expression data of microglia isolated after TAM-induced recombination of $C \times 3 c r 7^{C r e E R T 2}: R 26-y f p, T g f b r 2^{f l / f l}$ mice $(n=6)$. TAM-induced $C \times 3 c r 7^{+/+}: R 26-y f p$, Tgfbr $2^{f l / f l}$ mice $(n=6)$ were used as controls displaying no significant changes in expression of microglia-specific genes in Tgfbr2-deficient microglia. c Heatmap analysis of RNAseq expression data showing differentially expressed genes in Tgfbr2-deficient microglia. d GO term enrichment analysis of biological process (GOTERM_BP), e molecular function (GOTERM_MF) and $\mathbf{f}$ KEGG pathway enrichment analysis was performed using DAVID Bioinformatics Resources 6.8. $\mathbf{g}$ Scheme for microglia cellular compartment enrichment analysis is depicted with individually regulated compartment-associated genes 
a
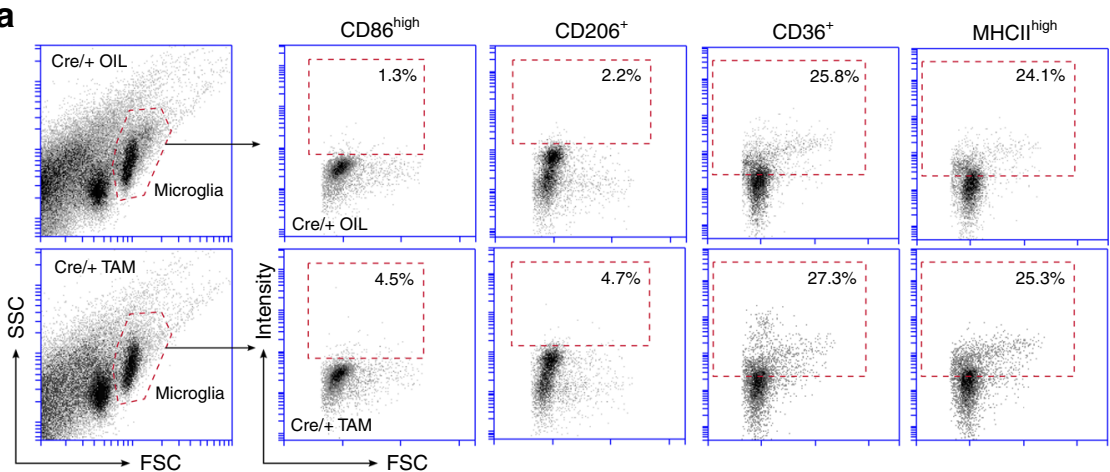

b
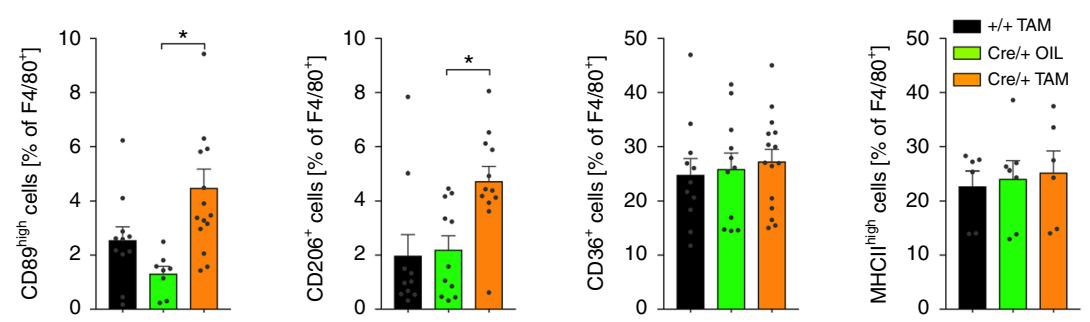

C
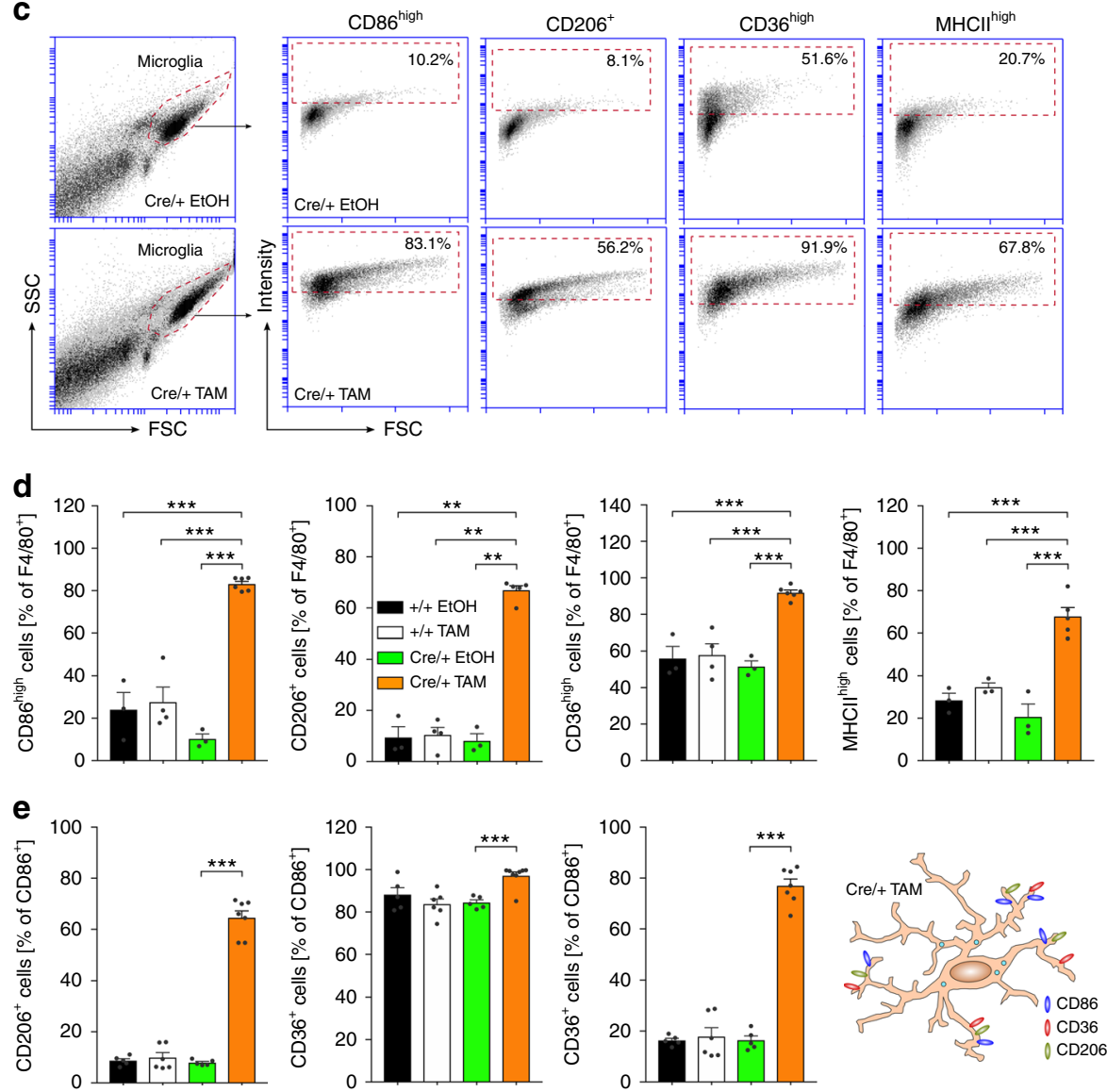

postnatal wasting syndrome due to uncontrolled systemic inflammatory responses ${ }^{19}$, Butovsky et al. ${ }^{12}$ reconstituted peripheral TGF $\beta 1$ expression under the control of the Il2 promoter resulting in the survival of mice into adulthood. This CNSspecific loss of TGF $\beta 1$ lead to a microglia phenotype characterised by loss of microglia-specific gene expression patterns and impaired microglia survival starting from postnatal day $20^{12}$. Among the genes being affected in microglia from TGF $\beta 1$ mutant

mice, Sall1 has been recently reported to maintain microglia identity and their respective physiological properties ${ }^{32}$. Moreover, the authors used Sall1 ${ }^{\text {CreERT2 }}$ mice to specifically target TGF $\beta$ signalling in adult microglia and their results suggested that TGF $\beta$ signalling in adult microglia is rather essential for microglia quiescence than for microglia maintenance and survival ${ }^{32}$. Using C $x 3$ cr $1^{\text {CreERT2 } 2}$ mice, we were able to confirm these recent findings and further demonstrate that mice undergo normal aging with 
Fig. 5 Analysis of microglial activation marker after TAM-induced deletion of Tgfbr2 in vivo and in vitro. a Flow cytometry analysis of microglia activation markers CD86, CD206, CD36, and MHCII after TAM-induced recombination in vivo. Representative flow cytometry graphs are shown for microglia from Cre/+ OIL control (top) and Cre/+ TAM knockout (bottom) Cx3cr7CreERT2:R26-yfp,Tgfbr fl/fl mice. b Quantifications of CD86 high, CD206 ${ }^{+}, \mathrm{CD}^{\mathrm{fl}} 6^{+}$and $\mathrm{MHCI} \mathrm{h}_{\text {high }}$ microglia $\left(\mathrm{F} 4 / 80^{+}\right)$are depicted. Data are presented as means \pm SEM of at least six independent experiments. $p$ values derived from one-way ANOVA are ${ }^{*} p<0.05$. c Flow cytometry analysis of microglia activation markers CD86, CD206, CD36 and MHCII 5 days after TAM-induced recombination in vitro. Representative flow cytometry graphs are shown for Cx3cr7CreERT2:R26-yfp,Tgfbr2 fl/fl microglia after treatment with EtOH (Cre/+ $\mathrm{EtOH}$, top) and TAM (Cre/+ TAM, bottom). d Quantifications of CD86 high, CD206+, CD36 high and MHCIligh microglia (F4/80+) are presented. e Quantifications of $\mathrm{CD} 6^{+} / \mathrm{CD} 206^{+}, \mathrm{CD} 6^{+} / \mathrm{CD} 36^{+}$and $\mathrm{CD} 206^{+} / \mathrm{CD} 36^{+}$double-positive microglia demonstrating the presence of all markers on the majority of $\mathrm{F} 4 / 80^{+}$Tgfbr2-deficient microglia $(\mathrm{Cre} /+\mathrm{TAM})$ in vitro. Data are presented as means $\pm \mathrm{SEM}$ of at least 63 independent experiments. $p$ values derived from one-way ANOVA are ${ }^{\star} p<0.05,{ }^{\star \star} p<0.01$ and ${ }^{\star \star \star} p<0.001$

regular weight gain showing unaffected microglia numbers. Using RNA-seq we were able to show that expression of microgliaspecific genes was not impaired after silencing TGF $\beta$ signalling in vivo. However, in vitro studies using P0 primary microglia have clearly demonstrated that TGF $\beta 1$ regulates the expression of these microglia-specific genes (Supplementary Fig. 5D). Taken into consideration that microglia maturation-as reflected by induction and expression of microglia-specific genes ${ }^{11}$ - peaks around P14, it is highly likely that TGF $\beta$ signalling between P0 and P14 plays a major role for this maturation process. Interestingly, we have recently demonstrated that TGF $\beta$ signalling peaks in microglia at $\mathrm{P} 7$, which precedes to upregulation of microglia-specific markers ${ }^{33}$. In the present study, male mice have been exclusively used to silence TGF $\beta$ signalling. Noteworthy, a sex dimorphism of postnatal microglia development has been described in mice ${ }^{34}$ and thus, female mice need to be included in future studies to address whether Tgfbr2-deficiency results in the same phenotype in female mice. Our data further demonstrate that abrogation of TGF $\beta$ signalling in mature microglia did not result in a loss of microglia identity indicating that TGF $\beta$ is dispensable for maintenance of mature microglia. However, the molecular mechanisms and the discrimination between direct TGF $\beta 1$ target genes and genes being regulated by secondary effects remain elusive and need further evaluation in order to understand how TGF $\beta 1$ contributes to microglia maturation.

Despite the fact that microglia maturation was not impaired after silencing TGF $\beta$ signalling, we clearly demonstrated that microglia displayed an activated and primed phenotype in vivo and in vitro. Holtman et al. ${ }^{35}$ have recently identified transcriptional profiles to distinguish between acutely activated, primed and disease-associated microglia. Comparison of these gene expression profiles with the signature observed in Tgfbr2-deficient microglia revealed that lack of TGF $\beta$ signalling resulted in upregulation of markers found in primed, aged, and immuneactivated microglia. Among the markers suggesting microglia activation, $C d 74$ and $C d 52$ were upregulated in Tgfbr2-deficient microglia in vivo. Interestingly, treatment of microglia with recombinant TGF $\beta 1$ in vitro resulted in strong downregulation of Cd74 and Cd52 (Supplementary Fig. 4B). Both CD74 36,37 and CD52 38 have been reported to be upregulated in activated microglia in neuropathologic conditions. CD74, also known as the MHC class II invariant chain, acts as an MHCII chaperone and indicates increased antigen presentation. Moreover, CD74 has been identified as the receptor for MIF (macrophage migration inhibitory factor) triggering activation of ERK, MAPK, and $\mathrm{Nf} \kappa \mathrm{B}$ signalling and, thus, increasing the release of proinflammatory cytokines thereby fostering immunological reactions $^{39}$. Interestingly, the small glycoprotein CD52 has recently been shown to limit $\mathrm{Nf} \kappa \mathrm{B}$ signalling in macrophages resulting in inhibition of proinflammatory cytokine production ${ }^{40}$. Moreover, $C y b b$, also referred to as Nox2 or gp91phox and Tnf were significantly upregulated after the abrogation of TGF $\beta$ signalling in microglia. Both candidates have been extensively demonstrated to be involved in microglia-mediated neuroinflammation and neurodegeneration ${ }^{41,42}$. However, although several upregulated genes suggest microglia activation, several genes which have been shown to exert anti-inflammatory effects also display increased expression. Wfdc17 as a counter-regulator of activated microglia $^{43}, M s 4 a 4 a$ recently described as a novel M2-like marker in macrophages $^{44}$, Aoah as a scavenger clearing and catabolizing free lipopolysaccharide ${ }^{45}$, or $M r c 1$ as one of the most prominent M2-like markers ${ }^{46}$ indicate that $T g f b r 2$-deficient microglia also display anti-inflammatory properties. It is likely that these genes might be upregulated in order to compensate and counteract microglia activation and it is further unclear which of the affected genes are direct TGF $\beta$ target genes in microglia. Although some of these genes are downregulated after treatment of microglia with recombinant TGF $\beta 1$ (Supplementary Figures 4B and 5B), a thorough study identifying TGF $\beta$ target genes will gain our understanding of the TGF $\beta 1$-mediated regulation of microglia activation states. It remains further to be evaluated whether the observed microglia activation in $C \times 3 c r 1^{C r e E R T 2}: R 26-y f p, T g f b r 2^{f l f f l}$ mice impairs neuronal survival and/or functions. Numbers of cortical NeuN ${ }^{+}$neurons in CX3cr1 CreERT2:R26-yfp,Tgfbr $2^{f l / f l}$ mice were not altered compared to control mice (Fig. $2 \mathrm{~h}$ ), but effects on distinct neuron subpopulations and possible impairments of their functional properties especially during aging of mice remain to be addressed.

The involvement of TGF $\beta 1$ in the regulation of microglia activation and, thus, mediating resolution of neuroinflammation and promoting neuroprotection has been demonstrated in models for neurodegenerative diseases including $\mathrm{AD}^{47,48}$ and $\mathrm{PD}^{49}$. Moreover, recent reports indicate that TGF $\beta 1$ is secreted by distinct cell populations such as astrocytes and mesenchymal stem cells to modify microglia functions ${ }^{50,51}$. However, the crosstalk between different CNS cells via TGF $\beta$ secretion and subsequent paracrine and/or autocrine effects is only partially understood. Whereas neurons are the major source of TGF $\beta 1$ under physiological conditions, microglia and to a lesser extent astrocytes increase expression of TGF $\beta 1$ in a middle cerebral artery occlusion model ${ }^{52}$. Interestingly, the levels of microglial Tgfbr 1 and Tgfbr 2 expression seem to be very low under basal conditions and rapidly increase in the same ischaemia model ${ }^{53}$. These observations indicate that microglia are more sensitive to TGF $\beta 1$ during their reactive states, thus, resulting in temporal and spatial limitations of TGF $\beta 1$-mediated effects on microglia. Microglia-specific conditional gene targeting has been a challenge in the past and attempts to silence TGF $\beta$ signalling using LysM ${ }^{C r e}, T g f b r 2^{f l f l}$ mice resulted in targeted deletion in monocyte-derived cells but not in CNS resident microglia 54 Using $C \times 3 c r 1^{C r e E R T 2}: R 26-y f p, T g f b r 2^{f l / f l}$ mice, we have clearly demonstrated an inducible microglia-specific deletion of Tgfbr2 and the fact that mutant mice do not show impaired survival and microglia maintenance offers the opportunity to study microglial TGF $\beta$ signalling in a variety of neurodegenerative disease models. 
a

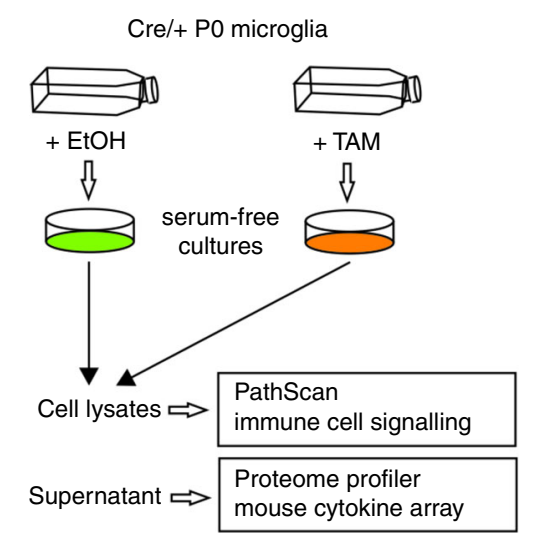

b

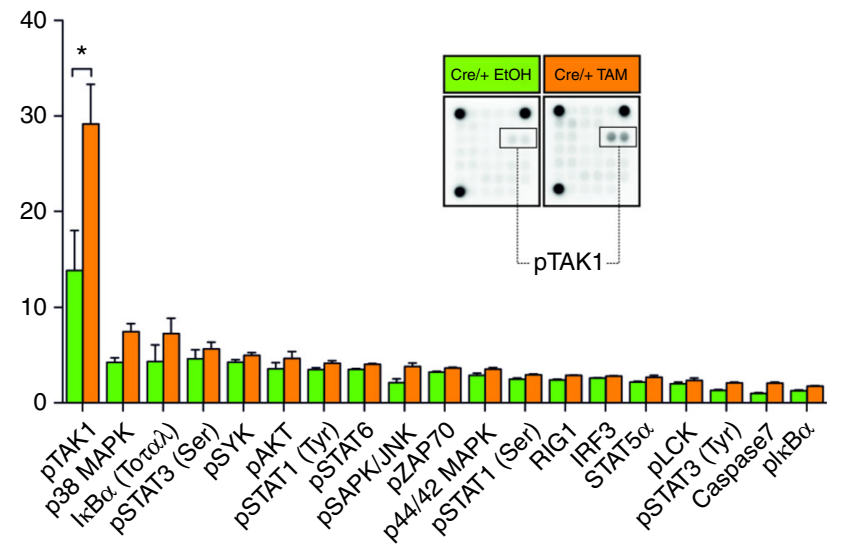

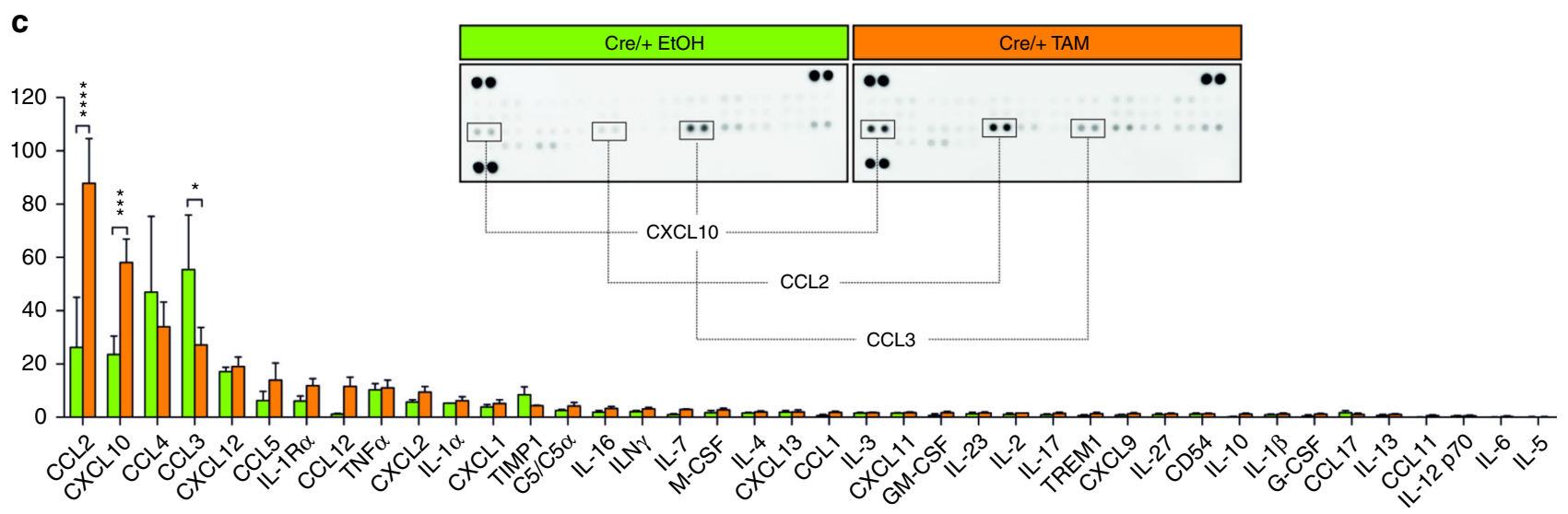

d

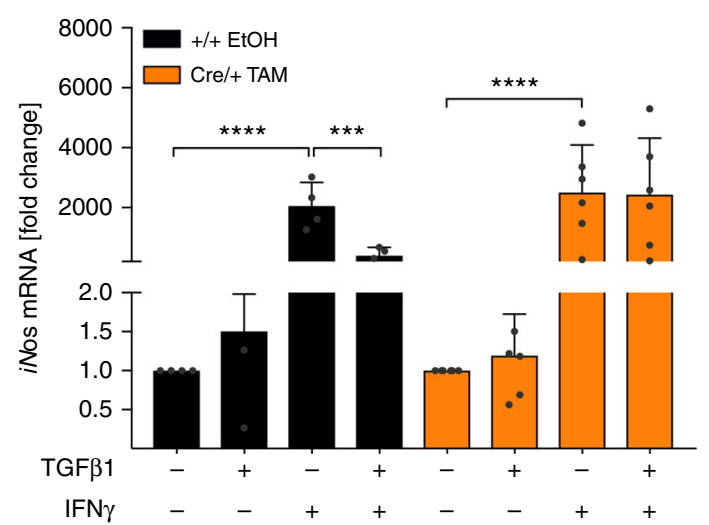

e

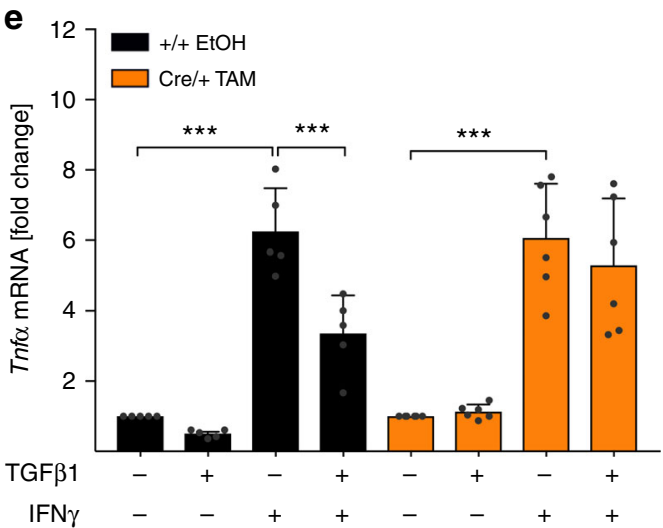

Fig. 6 Microglial-specific knockout of Tgfbr2 leads to increased TAK1 phosphorylation and increased CXCL10 and CCL2 secretion. a Primary microglia from

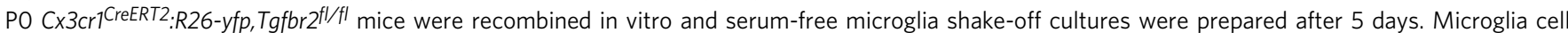
lysates were used for the detection of activated signalling proteins using the CST PathScan ${ }^{\circledR}$ Immune Cell Signaling Antibody Array Kit and supernatants of control $(\mathrm{Cre} /+\mathrm{EtOH})$ and knockout (Cre/+ TAM) microglia were used for cytokine and chemokine detection using the Proteome Profiler Mouse Cytokine Array. b Densitometric spot analysis of array membranes revealed increased TAK1 phosphorylation in Cre/+ TAM microglia. Data are presented as means \pm SEM from eight independent experiments. $p$ values derived from one-sample $t$ tests are ${ }^{\star} p<0.05$. c Quantification of cytokine secretion was performed by densitometric spot analysis of array membranes. Data are presented as means \pm SEM from three individual experiments. $p$ values derived from onesample $t$ tests are ${ }^{\star} p<0.05,{ }^{\star \star *} p<0.001$ and ${ }^{\star \star \star \star} p<0.0001$. Primary microglia from Cx3crlCreERT2:R26-yfp, Tgfbr $2^{f l / f l}$ mice were recombined in vitro, stimulated with TGF $\beta 1(5 \mathrm{ng} / \mathrm{ml})$ for $2 \mathrm{~h}$ and subsequently with IFN $\gamma(10 \mathrm{ng} / \mathrm{ml})$ or TGF $\beta 1+\mathrm{IFN} \gamma$ for $24 \mathrm{~h}$. mRNA levels of iNos (d) and Tnfa (e) values from quantitative RT-PCR were normalised to Gapdh. Graphs display fold changes compared to unstimulated samples of $+/+$ EtOH control samples and $\mathrm{cre} /+\mathrm{TAM}$ knockout samples, respectively. Data are presented as means $\pm \mathrm{SEM}(+/+\mathrm{EtOH} n=4-5, \mathrm{Cre} /+\mathrm{TAM} n=5-6) . p$ values derived from oneway ANOVA are ${ }^{\star \star \star} p<0.001$ and ${ }^{\star \star \star \star} p<0.0001$ 
Moreover, TGF $\beta$ signalling in microglia can be further analysed at different postnatal time points including aging where impaired microglial TGF $\beta$ signalling has been suggested to be linked with age-dependent microglia activation ${ }^{55}$. Taken together, the present study underlines the importance of TGF $\beta$ signalling for the regulation of microglia activation in vivo and in vitro and further introduces $C x 3 c r 1^{C r e E R T 2}: R 26-y f p$, Tgfbr fllfl $^{l}$ mice as a powerful tool to study TGF $\beta$-mediated effects during physiological and pathological functions of microglia in the CNS.

\section{Methods}

Mice. The generation of $\mathrm{C} x 3 \mathrm{cr} 1^{\mathrm{CreERT} 2}$ mice has been described recently ${ }^{22}$. $\mathrm{C} x 3 \mathrm{cr} 1^{\mathrm{CreERT} 2}$ mice were genotyped by PCR using the forward primer $5^{\prime}$-CCT CTA AGA CTC ACG TGG ACC TG-3', the reverse primer 5'-GAC TTC CGA GTT GCG GAG CAC-3' and specification primer 5'-GCC GCC CAC GAC CGG CAA AC-3' which amplify a 304 bp fragment from the transgenic $C x 3 c r 1$ locus. Mice carrying loxP-site-flanked (floxed) alleles of the Tgfbr 2 gen ${ }^{56}$ were crossed to the $C x 3 c r 1^{\text {CreERT2 }}$ line. R26-yfp mice (B6.129×1-Gt(ROSA)26Sortm1(EYFP)Cos/J) ${ }^{57}$ were additionally crossed into the $C \times 3{ }^{c r} 1^{\mathrm{CreERT2}}: \mathrm{Tgfbr} 2^{f l f l}$ mouse line. For induction of Cre recombinase activity, 6-8-week-old $\mathrm{C} x 3 \mathrm{cr} 1$ CreERT2 mice were treated with $8 \mathrm{mg}$ tamoxifen (TAM, T5648, Sigma-Aldrich, Germany) solved in $200 \mu \mathrm{l}$ corn oil (C8267, Sigma) injected subcutaneously and intraperitoneally at two time points $48 \mathrm{~h}$ apart. For all experiments, littermates carrying the respective loxPflanked alleles but lacking expression of Cre recombinase $(+/+$ TAM) or not receiving tamoxifen (cre/+ OIL) were used as controls. Male mice have been used throughout the study. All animal experiments were approved by the Federal Ministry for Nature, Environment and Consumers' Protection of the state of Baden-Württemberg and were performed in accordance to the respective national, federal and institutional regulations.

Microglia isolation. Mice were deeply anaesthetised by an intraperitoneal injection of ketamine $(75 \mathrm{mg} / \mathrm{kg}$ ) and Rompun $(5.8 \mathrm{mg} / \mathrm{kg}$ ) and perfused with ice-cold phosphate-buffered saline (PBS). The brains were removed from the skulls and meninges were removed by rolling over absorbent paper. Brains were collected in cold dissection buffer, homogenised with a glass homogeniser and filtered through $75 \mu \mathrm{m}$ cell strainers. Cells were washed with $30 \mathrm{ml}$ HBSS and centrifuged $12 \mathrm{~min}, 300 \times g, 4^{\circ} \mathrm{C}$. For density gradient centrifugation, the pellet was re-suspended in $5 \mathrm{ml} \mathrm{37 \%} \mathrm{Percoll} \mathrm{(P1644,} \mathrm{Sigma-Aldrich)} \mathrm{in} \mathrm{PBS,} \mathrm{underlaid}$ with $4 \mathrm{ml} 70 \%$ Percoll and overlaid with $4 \mathrm{ml} 30 \%$ Percoll in a $15 \mathrm{ml}$ Falcon Tube. The gradient was centrifuged for $40 \mathrm{~min}, 600 \times g, 15^{\circ} \mathrm{C}$, without breaks. Afterward, the cell layer was transferred to PBS $+1 \%$ FCS and centrifuged for 5 min, $200 \times g, 4^{\circ} \mathrm{C}$.

Primary cultures. Cells were prepared from newborn mice as described previously ${ }^{15}$. For induction of Cre recombinase activity, OH-TAM (H7904, SigmaAldrich, Germany) was applied at a final concentration of $1 \mu \mathrm{M}$ at least 3 days before analysis. Microglia were kept in serum-free medium for $2 \mathrm{~h}$, stimulated with TGF $\beta 1(5 \mathrm{ng} / \mathrm{ml})$ for $2 \mathrm{~h}$ and either subsequently fixed in $4 \%$ paraformaldehyde (PFA) and processed for fluorescence microscopy or harvested in PBS for gene expression and protein analysis.

Fluorescence microscopy. After transcardial perfusion with PBS and 4\% PFA, brains were post-fixed in 4\% PFA for additional $24 \mathrm{~h}$, embedded in $5 \%$ agarose, and $50 \mu \mathrm{m}$ vibratome sections were prepared. Cultured cells were fixed with $4 \%$ PFA. Sections and cultured cells were then blocked with PBS containing $5 \%$ bovine serum albumin and permeabilized with $0.1 \%$ Triton X-100. Primary antibodies were added overnight at a dilution of 1:500 for anti-Ibal (019-19741, WACO, Japan), 1:1000 for anti-GFP (600-106-215, Rockland Immunochemicals Inc., Gilbertsville, USA), 1:200 for anti-SMAD1/2/3 (sc-7960, Santa Cruz) and 1:200 for anti-SMAD4 (sc-7966, Santa Cruz) at $4{ }^{\circ} \mathrm{C}$. Secondary antibodies were added as follows: Alexa Fluor 488 and Alexa Fluor 568, 1:500, for $2 \mathrm{~h}$ at room temperature. Nuclei were counterstained using DAPI. Ibal ${ }^{+}$microglia were counted in at least three cortical areas $(500 \times 500 \mu \mathrm{m})$ of each animal in the maximum intensity projection of $50 \mu \mathrm{m}$ thickness. In cultured cells, SMAD1/2/3 ${ }^{+}$and SMAD4 ${ }^{+}$ microglia were counted in three visual fields at $\times 20$ magnification. SMAD ${ }^{+}$cells were set in relation to all $\mathrm{DAPI}^{+}$cell. The number of cells and the examined areas were determined using a Zeiss AxioImage M2 microscope (ZEISS, Göttingen, Germany).

Three-dimensional reconstruction of microglia. Free-floating $50-\mu \mathrm{m}$ vibratome sections from adult brain tissue were stained overnight with anti-IBA1 (1:500) at 4 ${ }^{\circ} \mathrm{C}$, followed by Alexa Fluor 568-conjugated secondary antibody at a dilution of 1:500 for $2 \mathrm{~h}$ at $20-25^{\circ} \mathrm{C}$. Nuclei were counterstained with DAPI. Imaging was performed on a Leica TCS SP8 confocal laser scanning microscope with a $\times 20$ oil immersion objective and the LAS AF image analysis software. $Z$-stacks with $1.1-\mu \mathrm{m}$ steps in the $z$ direction, $1024 \times 1024$ pixel resolution, were analysed using Imaris software (Bitplane). Microglia from cortices (layers 2-5) corresponding to bregma levels -2 to -4 were used for the analysis.

Flow cytometry. Cells were stained with primary antibodies directed against CD11b (1:20, 53-0112-82, eBioscience), CD206 (5 $\mu$ l, FAB2535C, R\&D Systems), CD36 (5 $\mu$ l, MCA2748A647, AbD Serotech), CD45 (1:20, 17-0451-82, eBioscience), CD86 (5 $\mu$ l, MCA2463PE, AbD Serotech), F4/80 (5 $\mu$ l, MCA497A488, AbD Serotech), MHCII (10 $\mu$ l, MCA2401P647, AbD Serotech) at $4{ }^{\circ} \mathrm{C}$ for $15 \mathrm{~min}$. An Fc receptor blocking antibody (TrueStain $\mathrm{fcX}, 101319$, Biolegend) was used to avoid unspecific antibody binding. Cells were washed and analysed using a BD Accuri C6 flow cytometer or sorted by BD Cell Sorter FACS Aria Fusion and BD Cell Sorter FACS Aria III.

qRT-PCR. RNA of microglia was isolated using PicoPure ${ }^{\text {ma }}$ RNA Isolation Kit (KIT0202, Arcturus) according to the manufacturer's protocol. Samples were treated with DNaseI (79254, Qiagen) and RNA was transcribed into cDNA using High Capacity RNA-to-cDNA Kit (4387406, Life Technologies). Five microlitres cDNA was transferred into a 96-well Multiply PCR plate (MLL 9601, Biorad) with $15 \mu \mathrm{l} \mathrm{GoTaq}{ }^{\circledast}$ qPCR Master Mix (A6002, Promega). RT-PCR reactions were performed as described recently ${ }^{33}$. Primers Tgfbr2for $5^{\prime}$-TAA-

CAGTGATGTCATGGCCAGCG-3', Tgfbr2rev $5^{\prime}$ -

AGACTTCATGCGGCTTCTCACAGA-3', Gapdhfor 5'-ATGACTCTACCCACGGCAAG-3', Gapdhrev 5'-GATCTCGCTCCTGGAAGATG-3', iNosfor 5'CAAGAGTTTGACCAGAGGACC-3', iNosrev 5'-TGGAACCACTCGTACTTGGGA-3', Tnfofor 5'-GACCCTCACACTCAGATCAT-3', Tnforev 5'TTGAAGAGAACCTGGGAGTA- $3^{\prime}$.

Western blot analysis. Tissues or cells were extracted in RIPA buffer (\#9806, New England Biolabs). Samples were separated by SDS-PAGE and immunoblotted using antibodies to pSMAD2 (1:500, 3101s, Cell Signaling) and GAPDH (1:100, 2118, Cell Signaling). Quantification was performed using ImageJ software. Uncropped scans of the blots are presented in Supplementary Fig. 2D.

Protein arrays. Primary microglia were counted and plated with equal numbers on $3 \mathrm{~cm}$ cell culture dishes for $24 \mathrm{~h}$. The cells were then washed once with PBS and given serum-free medium. After $24 \mathrm{~h}$, cells were lysed and used for the PathScan ${ }^{\circledR}$ Immune Cell Signalling Antibody Array Kit (13792, Cell Signalling Technologies) and the supernatant was used for Proteome Profiler Mouse Cytokine Array Kit (ARY006, R\&D) according to the manufacturer's protocols.

RNA Seq. Total RNA was extracted from FACS sorted microglia cells using Picopure RNA extraction kit (Life Technologies) according to the manufacturer's protocol. Isolated RNA was controlled for quantity and determination of an RNA integrity score (RIN) using RNA pico chips on a Bioanalyzer 2100 (Agilent). Sample preparation for microarray hybridisation was carried out as described in the NuGEN Ovation Pico WTA System V2 and NUGEN Encore Biotin Module manuals (NuGEN Technologies, Inc, San Carlos, CA, USA). In brief, between 0.4 and $2.5 \mathrm{ng}$ of total RNA was reverse transcribed into double-stranded cDNA in a two-step process, introducing an SPIA tag sequence. Bead purified cDNA was amplified by an SPIA amplification reaction followed by an additional bead purification. $3.0 \mu \mathrm{g}$ of SPIA cDNA was fragmented, terminally biotin-labelled and hybridised to an Affymetrix Mouse Gene 2.0 ST Array Plate. For hybridisation, washing, staining and scanning an Affymetrix GeneTitan system, controlled by the Affymetrix GeneChip Command Console software v4.2, was used. Sample processing was performed at an Affymetrix Service Provider and Core Facility, "KFBCenter of Excellence for Fluorescent Bioanalytics" (Regensburg, Germany; www. kfb-regensburg.de). Differential Gene Expression Analyses were performed using DAVID $^{24}$.

Statistics. All statistical analysis was performed using GraphPad Prism 6. Values are expressed as means \pm standard error of the mean (SEM). Significance was assessed using a $95 \%$ confidence level. $G^{*}$ Power 3.1 (University Düsseldorf, Germany) was used to determine sample sizes. Unpaired, two-tailed, parametric $t$ tests were used for comparison of two sets of absolute values. "One-sample $t$ tests" are used for values expressed as fold changes, where the analysed value was compared to the hypothetical value 1. One-way ANOVA with correction for multiple comparisons was used for three or more sets of absolute values. Tukey multiple comparison test was used to compare all groups with each other. Two-way ANOVA was used in protein arrays to consider both changed and overall protein levels.

\section{Data availability}

RNAseq as well as microarray data have been deposited to NCBI GEO and are available as accession numbers GSE115652 (TGF $\beta 1$-treated primary microglia, microarray data) and GSE115757 (Tgfbr2-deficient microglia, sorted, RNAseq). The data that support the findings of this study are available from the corresponding author upon reasonable request. 
Received: 1 November 2017 Accepted: 20 August 2018

Published online: 01 October 2018

\section{References}

1. Prinz, M., Priller, J., Sisodia, S. S. \& Ransohoff, R. M. Heterogeneity of CNS myeloid cells and their roles in neurodegeneration. Nat. Neurosci. 14, 1227-1235 (2011).

2. Prinz, M. \& Priller, J. Microglia and brain macrophages in the molecular age: from origin to neuropsychiatric disease. Nat. Rev. Neurosci. 15, 300-312 (2014).

3. Wolf, S. A., Boddeke, H. W. G. M. \& Kettenmann, H. Microglia in physiology and disease. Annu. Rev. Physiol. 79, 619-643 (2017).

4. Perry, V. H. \& Holmes, C. Microglial priming in neurodegenerative disease. Nat. Rev. Neurol. 10, 217-224 (2014).

5. Spittau, B. Aging microglia-phenotypes, functions and implications for age-related neurodegenerative diseases. Front. Aging Neurosci. 9, 194 (2017).

6. Ginhoux, F. et al. Fate mapping analysis reveals that adult microglia derive from primitive macrophages. Science 330, 841-845 (2010).

7. Kierdorf, K. et al. Microglia emerge from erythromyeloid precursors via Pu.1and Irf8-dependent pathways. Nat. Neurosci. 16, 273-280 (2013).

8. Greter, M. et al. Stroma-derived interleukin-34 controls the development and maintenance of langerhans cells and the maintenance of microglia. Immunity 37, 1050-1060 (2012).

9. Beutner, C. et al. Unique transcriptome signature of mouse microglia. Glia 61, 1429-1442 (2013).

10. Hickman, S. E. et al. The microglial sensome revealed by direct RNA sequencing. Nat. Neurosci. 16, 1896-1905 (2013).

11. Bennett, M. L. et al. New tools for studying microglia in the mouse and human CNS. Proc. Natl. Acad. Sci. USA 113, E1738-1746 (2016).

12. Butovsky, O. et al. Identification of a unique TGF- $\beta$-dependent molecular and functional signature in microglia. Nat. Neurosci. 17, 131-143 (2014).

13. Brionne, T. C., Tesseur, I., Masliah, E. \& Wyss-Coray, T. Loss of TGF-beta 1 leads to increased neuronal cell death and microgliosis in mouse brain. Neuron 40, 1133-1145 (2003).

14. Makwana, M. et al. Endogenous transforming growth factor beta 1 suppresses inflammation and promotes survival in adult CNS. J. Neurosci. 27, 11201-11213 (2007)

15. Spittau, B. et al. Endogenous transforming growth factor-beta promotes quiescence of primary microglia in vitro. Glia 61, 287-300 (2013).

16. Wrana, J. L., Attisano, L., Wieser, R., Ventura, F. \& Massagué, J. Mechanism of activation of the TGF-beta receptor. Nature 370, 341-347 (1994).

17. Yamashita, H., ten Dijke, P., Franzén, P., Miyazono, K. \& Heldin, C. H. Formation of hetero-oligomeric complexes of type I and type II receptors for transforming growth factor-beta. J. Biol. Chem. 269, 20172-20178 (1994).

18. Massagué, J. \& Wotton, D. Transcriptional control by the TGF-beta/Smad signaling system. EMBO J. 19, 1745-1754 (2000).

19. Shull, M. M. et al. Targeted disruption of the mouse transforming growth factor-beta 1 gene results in multifocal inflammatory disease. Nature 359, 693-699 (1992).

20. Oshima, M., Oshima, H. \& Taketo, M. M. TGF-beta receptor type II deficiency results in defects of yolk sac hematopoiesis and vasculogenesis. Dev. Biol. 179, 297-302 (1996).

21. Takaku, K. et al. Intestinal tumorigenesis in compound mutant mice of both Dpc4 (Smad4) and Apc genes. Cell 92, 645-656 (1998).

22. Goldmann, T. et al. A new type of microglia gene targeting shows TAK1 to be pivotal in CNS autoimmune inflammation. Nat. Neurosci. 16, 1618-1626 (2013).

23. Harry, G. J. Microglia during development and aging. Pharmacol. Ther. 139, 313-326 (2013).

24. Dennis, G. et al. DAVID: Database for Annotation, Visualization, and Integrated Discovery. Genome Biol. 4, P3 (2003).

25. Zhou, X., Zöller, T., Krieglstein, K. \& Spittau, B. TGF $\beta 1$ inhibits IFN $\gamma$ mediated microglia activation and protects $\mathrm{mDA}$ neurons from IFN $\gamma$-driven neurotoxicity. J. Neurochem. 134, 125-134 (2015).

26. Machado, V., Zöller, T., Attaai, A. \& Spittau, B. Microglia-mediated neuroinflammation and neurotrophic factor-induced protection in the MPTP mouse model of Parkinson's disease-lessons from transgenic mice. Int. J. Mol. Sci. 17, E151 (2016).

27. Stevens, B. et al. The classical complement cascade mediates CNS synapse elimination. Cell 131, 1164-1178 (2007).

28. Paolicelli, R. C. et al. Synaptic pruning by microglia is necessary for normal brain development. Science 333, 1456-1458 (2011).
29. Parkhurst, C. N. et al. Microglia promote learning-dependent synapse formation through brain-derived neurotrophic factor. Cell 155, 1596-1609 (2013).

30. Squarzoni, P. et al. Microglia modulate wiring of the embryonic forebrain. Cell Rep. 8, 1271-1279 (2014).

31. Ueno, $\mathrm{M}$. et al. Layer $\mathrm{V}$ cortical neurons require microglial support for survival during postnatal development. Nat. Neurosci. 16, 543-551 (2013).

32. Buttgereit, A. et al. Sall 1 is a transcriptional regulator defining microglia identity and function. Nat. Immunol. 17, 1397-1406 (2016).

33. Attaai, A. et al. Postnatal maturation of microglia is associated with alternative activation and activated TGF $\beta$ signaling. Glia https://doi.org/10.1002/ glia.23332 (2018).

34. Weinhard, L. et al. Sexual dimorphism of microglia and synapses during mouse postnatal development. Dev. Neurobiol. 78, 618-626 (2018).

35. Holtman, I. R. et al. Induction of a common microglia gene expression signature by aging and neurodegenerative conditions: a co-expression metaanalysis. Acta Neuropathol. Commun. 3, 31 (2015).

36. Zeiner, P. S. et al. MIF receptor CD74 is restricted to microglia/macrophages, associated with a M1-polarized immune milieu and prolonged patient survival in gliomas. Brain Pathol. 25, 491-504 (2015).

37. Wang, J. et al. CD74 indicates microglial activation in experimental diabetic retinopathy and exogenous methylglyoxal mimics the response in normoglycemic retina. Acta Diabetol. 51, 813-821 (2014).

38. Chatterjee, D. et al. Mouse hepatitis virus infection upregulates genes involved in innate immune responses. PLoS ONE 9, e111351 (2014).

39. Su, H., Na, N., Zhang, X. \& Zhao, Y. The biological function and significance of CD74 in immune diseases. Inflamm. Res. 66, 209-216 (2017).

40. Rashidi, M. et al. CD52 inhibits Toll-like receptor activation of NF- $\kappa B$ and triggers apoptosis to suppress inflammation. Cell Death Differ. 25, 392-405 (2018).

41. Chen, S.-H., Oyarzabal, E. A. \& Hong, J.-S. Critical role of the Mac1/NOX2 pathway in mediating reactive microgliosis-generated chronic neuroinflammation and progressive neurodegeneration. Curr. Opin. Pharmacol. 26, 54-60 (2016).

42. Probert, L. TNF and its receptors in the CNS: The essential, the desirable and the deleterious effects. Neuroscience 302, 2-22 (2015).

43. Karlstetter, M. et al. The novel activated microglia/macrophage WAP domain protein, AMWAP, acts as a counter-regulator of proinflammatory response. J. Immunol. 185, 3379-3390 (2010)

44. Sanyal, R. et al. MS4A4A: a novel cell surface marker for M2 macrophages and plasma cells. Immunol. Cell Biol. https://doi.org/10.1038/icb.2017.18 (2017).

45. Shao, B., Munford, R. S., Kitchens, R. \& Varley, A. W. Hepatic uptake and deacylation of the LPS in bloodborne LPS-lipoprotein complexes. Innate. Immun. 18, 825-833 (2012).

46. Gensel, J. C. \& Zhang, B. Macrophage activation and its role in repair and pathology after spinal cord injury. Brain Res. 1619, 1-11 (2015).

47. Caraci, F. et al. Dysfunction of TGF- $\beta 1$ signaling in Alzheimer's disease: perspectives for neuroprotection. Cell Tissue Res. 347, 291-301 (2012).

48. Chen, J.-H., Ke, K.-F., Lu, J.-H., Qiu, Y.-H. \& Peng, Y.-P. Protection of TGF$\beta 1$ against neuroinflammation and neurodegeneration in $A \beta 1-42$-induced Alzheimer's disease model rats. PLoS ONE 10, e0116549 (2015).

49. Chen, X., Liu, Z., Cao, B.-B., Qiu, Y.-H. \& Peng, Y.-P. TGF- $\beta 1$ neuroprotection via inhibition of microglial activation in a rat model of Parkinson's disease. J. Neuroimmune. Pharmacol. 12, 433-446 (2017).

50. Norden, D. M., Fenn, A. M., Dugan, A. \& Godbout, J. P. TGF $\beta$ produced by IL-10 redirected astrocytes attenuates microglial activation. Glia 62, 881-895 (2014).

51. Noh, M. Y. et al. Mesenchymal stem cells modulate the functional properties of microglia via TGF- $\beta$ secretion. Stem Cells Transl. Med. 5, 1538-1549 (2016).

52. Pál, G. et al. Time course, distribution and cell types of induction of transforming growth factor betas following middle cerebral artery occlusion in the rat brain. PLoS ONE 7, e46731 (2012).

53. Pál, G., Lovas, G. \& Dobolyi, A. Induction of transforming growth factor beta receptors following focal ischemia in the rat brain. PLoS ONE 9, e106544 (2014).

54. Parsa, R. et al. TGF $\beta$ regulates persistent neuroinflammation by controlling Th1 polarization and ROS production via monocyte-derived dendritic cells. Glia 64, 1925-1937 (2016).

55. Tichauer, J. E. et al. Age-dependent changes on TGF $\beta 1$ Smad3 pathway modify the pattern of microglial cell activation. Brain Behav. Immun. https:// doi.org/10.1016/j.bbi.2013.12.018 (2013).

56. Chytil, A., Magnuson, M. A., Wright, C. V. E. \& Moses, H. L. Conditional inactivation of the TGF-beta type II receptor using Cre:Lox. Genesis 32, 73-75 (2002).

57. Srinivas, S. et al. Cre reporter strains produced by targeted insertion of EYFP and ECFP into the ROSA26 locus. Bmc Dev. Biol. 1, 4 (2001). 


\section{Acknowledgements}

This work was funded by grants from the Deutsche Forschungsgemeinschaft (DFG SP 51555/2-1). The authors thank Ludmila Butenko for her excellent technical assistance.

\section{Author contributions}

T.Z. and B.S. designed the study. T.Z., A.S., C.K., P.S.P., T.M., and B.S. performed experiments. D.P. processed RNA samples for cDNA microarrays. T.B. processed RNA samples for RNAseq. T.Z., M.P., and B.S. wrote the manuscript.

\section{Additional information}

Supplementary Information accompanies this paper at https://doi.org/10.1038/s41467018-06224-y.

Competing interests: The authors declare no competing interests.

Reprints and permission information is available online at http://npg.nature.com/ reprintsandpermissions/
Publisher's note: Springer Nature remains neutral with regard to jurisdictional claims in published maps and institutional affiliations.

(c) (1)

Open Access This article is licensed under a Creative Commons Attribution 4.0 International License, which permits use, sharing, adaptation, distribution and reproduction in any medium or format, as long as you give appropriate credit to the original author(s) and the source, provide a link to the Creative Commons license, and indicate if changes were made. The images or other third party material in this article are included in the article's Creative Commons license, unless indicated otherwise in a credit line to the material. If material is not included in the article's Creative Commons license and your intended use is not permitted by statutory regulation or exceeds the permitted use, you will need to obtain permission directly from the copyright holder. To view a copy of this license, visit http://creativecommons.org/ licenses/by/4.0/.

(C) The Author(s) 2018 